\title{
Comparative Study of Multifragmentation of Gold Nuclei Induced by Relativistic Protons, ${ }^{4} \mathrm{He}$, and ${ }^{12} \mathrm{C}$
}

\author{
S.P. Avdeyev ${ }^{a}$, V.A. Karnaukhov ${ }^{a}$, L.A. Petrov ${ }^{a \star}$, \\ V.K. Rodionov ${ }^{a}$, P.A. Rukoyatkin ${ }^{a}$, V.D. Toneev ${ }^{a}$, \\ H. Oeschler ${ }^{b \star \star}$, O.V. Bochkarev ${ }^{c}$, L.V. Chulkov ${ }^{c}$, \\ E.A. Kuzmin ${ }^{c}$, A. Budzanowski ${ }^{d}$, W. Karcz ${ }^{d}$, M. Janicki ${ }^{d}$, \\ E. Norbeck ${ }^{e}$, A.S. Botvina ${ }^{f}$, K.K. Gudima ${ }^{g}$ \\ a Joint Institute for Nuclear Research, 141980 Dubna, Russia \\ ${ }^{b}$ Institut für Kernphysik, Darmstadt University of Technology, 64289 Darmstadt, \\ Germany \\ ${ }^{c}$ Kurchatov Institute, 123182, Moscow, Russia \\ ${ }^{d}$ H. Niewodniczanski Institute of Nuclear Physics, 31-342, Cracow, Poland \\ e University of Iowa, Iowa City, IA 52242 USA \\ ${ }^{f}$ Institute for Nuclear Research, 117312, Moscow, Russia \\ ${ }^{g}$ Institute of Applied Physics, Kishinev, Moldova
}

\begin{abstract}
Multiple emission of intermediate-mass fragments has been studied for the collisions of $\mathrm{p},{ }^{4} \mathrm{He}$ and ${ }^{12} \mathrm{C}$ on $\mathrm{Au}$ with the $4 \pi$ setup FASA. The mean IMF multiplicities (for the events with at least one IMF) are saturating at the value of $2.2 \pm 0.2$ for the incident energies above $6 \mathrm{GeV}$. The observed IMF multiplicities cannot be described in a two-stage scenario, a fast cascade followed by a statistical multifragmentation. Agreement with the measured IMF multiplicities is obtained by introducing an intermediate phase and modifying empirically the excitation energies and masses of the remnants.

The angular distributions and energy spectra from the p-induced collisions are in agreement with the scenario of "thermal" multifragmentation of a hot and diluted target spectator. In the case of ${ }^{12} \mathrm{C}+\mathrm{Au}(22.4 \mathrm{GeV})$ and ${ }^{4} \mathrm{He}(14.6 \mathrm{GeV})+\mathrm{Au}$ collisions, deviations from a pure thermal break-up are seen in the energy spectra of the emitted fragments, which are harder than those both from model calculations and from the measured ones for p-induced collisions. This difference is attributed to a collective flow.
\end{abstract}

Key words: Nuclear Reactions; $\mathrm{p}(2-8 \mathrm{GeV}),{ }^{4} \mathrm{He}(4$ and $14.6 \mathrm{GeV}),{ }^{12} \mathrm{C}(22.4$ $\mathrm{GeV})+{ }^{197} \mathrm{Au}$; measured IMF multiplicity; charge, energy and angular

\footnotetext{
$\star$ deceased

${ }^{\star}$ Corresponding author: email: h.oeschler@gsi.de
} 
distributions; collective flow; comparison with the model calculations. 
PACS Numbers: 25.40-h, 25.70.Pq, 25.75-q

\section{Introduction}

Nuclear fragmentation was discovered in cosmic rays 60 years ago $[1,2]$ as a puzzling phenomenon in which nuclear fragments are emitted from collisions of relativistic protons with various targets. Fragments heavier than $\alpha$ particles but lighter than fission fragments have been observed. Now, they are commonly called Intermediate Mass Fragments (IMF, $3 \leq Z \leq 20$ ). Later on, in the fifties, this phenomenon was observed in accelerator experiments [3] and then studied leisurely for three decades. The situation changed dramatically in 1982 when multiple emission of IMF's was discovered in the ${ }^{12} \mathrm{C}$ (1030 MeV) irradiation of emulsion at the CERN synchrocyclotron [4]. These findings stimulated the development of many theoretical models to put forward the attractive idea that copious production of IMF's may be related to a liquid-gas phase transition in nuclear matter [5-9]. The recent status on multifragmentation can be found in Ref. [10].

About a dozen sophisticated experimental devices were created to investigate this process by using heavy ion beams, which are well suited for producing extremely hot systems. But in the case of heavy projectiles, nuclear heating is accompanied by compression, fast rotation and shape distortion which may cause dynamic effects in the multi-fragment disintegration, and it is not easy to disentangle all these effects and extract information on thermodynamic properties of hot nuclear systems. The situation becomes more transparent if light relativistic projectiles are used. In this case, dynamic effects are expected to be negligible. Another advantage is that all the fragments are emitted by a single source - a slowly moving target remainder. Its excitation energy should be almost entirely thermal. Light relativistic projectiles provide therefore a unique possibility for studying "thermal multifragmentation". It has been shown that thermal multifragmentation indeed takes place in collisions of light relativistic projectiles ( $\left.\mathrm{p}, \overline{\mathrm{p}},{ }^{3} \mathrm{He},{ }^{4} \mathrm{He}, \pi^{-}\right)$with a heavy target, and fragments are emitted from a diluted excited residue after an expansion driven by thermal pressure [11-16]. From IMF-IMF correlation data, fragment emission times of less than $100 \mathrm{fm} / \mathrm{c}$ have been deduced [17-19]. This value is considerably smaller than the characteristic Coulomb time $\tau_{c} \approx 10^{-21} \mathrm{~s}$ [20], which is the mean time for the Coulomb acceleration of fragments.

The time scale of the IMF emission is a crucial question for understanding this decay mode: Is it a "slow" sequential process of independent emission of IMF's or a new (multibody) decay mode with "simultaneous" ejection of fragments governed by the total accessible phase space? Only the latter process 
is usually called "multifragmentation". "Simultaneous" emission means that all fragments are liberated during a time smaller than the characteristic one $\tau_{c}$. In that case the IMF's are not emitted independently, as they interact via Coulomb forces and are accelerated after freeze out in a common electric field. Based on the measured emission times [17-19], the trivial mechanism of multiple IMF emission as independent fragment evaporation is excluded.

In this paper we present results of the experimental study of the multifragment emission induced by relativistic helium and carbon ions and compare them with our data [13] obtained for $\mathrm{p}+\mathrm{Au}$ collisions. The fragment multiplicities, energy, charge and angular distributions measured are analyzed in the framework of the combined approach: Cascade Model followed by the Statistical Multifragmentation Model. Emphasis is put on the question of thermalization and the study of a transition from a pure statistical process to a behavior evidencing the onset of a fast expansion as already demonstrated in [21].

\section{The Experiment}

\subsection{Experimental Setup}

The experiments were performed with beams from the JINR synchrophasotron in Dubna using the $4 \pi$-setup FASA. Details are given in $[22,23]$ including a brief description of the hardware and software. The device consists of two main parts : First, five $\Delta E$ (ionization chambers) $\times E(\mathrm{Si})$-telescopes, which serve as a trigger for the read-out of the system allowing measurement of the charge and energy distributions of IMF's at different angles. They are located at $\theta=24^{\circ}, 68^{\circ}, 87^{\circ}, 112^{\circ}$ and $156^{\circ}$ to the beam direction and together cover a solid angle of $0.03 \mathrm{sr}$. Second, the fragment multiplicity detector (FMD) consisting of $64 \mathrm{CsI}(\mathrm{Tl})$ counters (with thicknesses around $35 \mathrm{mg} \cdot \mathrm{cm}^{-2}$ ) which covers $89 \%$ of $4 \pi$. The FMD gives the number of IMF's in the event and their spatial distribution.

All the detectors have been calibrated with an alpha source $\left({ }^{241} \mathrm{Am}\right)$ located in the centre of vacuum chamber and precision-pulse generator. The accuracy in calibrating the energy scale is estimated to better than $5 \%$. This value is used as systematic error in the fragment-energy measurements.

A self-supporting Au target $1.5 \mathrm{mg} / \mathrm{cm}^{2}$ was located in the center of the FASA vacuum chamber ( $\sim 1 \mathrm{~m}$ in diameter $)$. The following beams were used: protons at energies of 2.16, 3.6 and $8.1 \mathrm{GeV},{ }^{4} \mathrm{He}$ at energies of 4 and $14.6 \mathrm{GeV}$ and ${ }^{12} \mathrm{C}$ at $22.4 \mathrm{GeV}$. The average beam intensity was $7 \cdot 10^{8} \mathrm{p} / \mathrm{spill}$ for protons and helium and $1 \cdot 10^{8} \mathrm{p} /$ spill for carbon projectiles with a spill length of 300 
ms and a spill period of $10 \mathrm{~s}$.

\subsection{Analysis of Fragment Multiplicity}

Using the FMD array, the associated IMF multiplicity distribution $W_{A}\left(M_{A}\right)$ is measured in events triggered by a fragment in at least one of the telescopes. The triggering probability is proportional to the multiplicity $M$ of the event (primary IMF multiplicity). Hence, the contribution of events with higher multiplicities in $W_{A}\left(M_{A}\right)$ is enhanced. This is a reason why $W_{A}\left(M_{A}\right)$ differs from the primary multiplicity distribution $W(M)$. Another reason is the FMD efficiency which is less than $100 \%$ and depends on the off-line threshold of the scintillator counters being adjusted in such a way to reduce the admixture of particles with $Z \leq 2$ in the counting rate of IMF's up to the level $\leq 5 \%$. These distributions are mutually related via the response matrix of the FASA setup $Q\left(M_{A}, M\right)$ (for details see Ref. [22,23]):

$$
W_{A}\left(M_{A}\right)=\sum_{M=M_{A}+1} Q\left(M_{A}, M\right) \cdot W(M)
$$

There are two options for obtaining the primary multiplicity distribution $W(M)$ from the measured one $W_{A}\left(M_{A}\right)$. The first is to parameterize the $W(M)$ distribution (using Fermi functions), to fold it with the experimental filter according to Eq. (1) and then to find the parameters by the best fit to the data. The second option is the direct reconstruction of $W(M)$ using the inverse matrix $Q^{-1}\left(M, M_{A}\right)$. Both procedures give rise to similar results.

In Fig. 1 the multiplicity distributions obtained for the gold target fragmentation by $14.6 \mathrm{GeV}$ alphas and $22.4 \mathrm{GeV}$ carbon ions are compared to that for the $\mathrm{p}(8.1 \mathrm{GeV})+\mathrm{Au}$ collision. In these cases the mean values $\langle M\rangle$ are always about $2.1-2.2$ (see Table 1 ) being close to that obtained by the ISIS group for ${ }^{3} \mathrm{He}+\mathrm{Au}$ collisions at $4.8 \mathrm{GeV}[24]$. Note, these values correspond to events with at least one IMF emitted. In this definition $M$ is never less than 1. The mean multiplicity for the all inelastic events is smaller by the factor $[1-P(0)]$ where $P(0)$ is the probability to have no IMF in the collision.

\section{Model Calculations}

The reaction mechanism of multifragmentation induced by light relativistic projectiles is usually divided into two steps [25]. The first one consists of a fast energy-deposition stage, during which very energetic light particles are

emitted and a nuclear remnant (spectator) is excited. The second one is a 
decay of the target spectator. The fast stage is usually described in terms of a kinetic approach. We use a refined version of the intranuclear cascade model [26] to get the distributions of nuclear remnants in charge, mass and excitation energy. The second stage can be described by multifragmentation models. The Statistical Multifragmentation Model (SMM) [27] coupled on the event-by-event basis to the cascade model is employed here. It will be discussed in section 4 whether the assumption of thermal equilibrium is justified.

\subsection{Refined Cascade Model}

The Refined Cascade Model (RC) is a version of the Quark-Gluon String Model developed in Ref. [28] and extended towards intermediate energies in Ref. [29]. This is a microscopic model which is based on the relativistic Boltzmann-type transport equations and the string phenomenology of hadronic interactions. Baryons and mesons belonging to the two lowest $S U(3)$ multiplets along with their antiparticles are included. The interactions between the hadrons are described by a collision term, where the Pauli principle is imposed in the final states. This includes elastic collisions as well as hadron production and resonance-decay processes. A fixed formation time $\tau_{f}=1$ $\mathrm{fm} / \mathrm{c}$ for produced particles is incorporated. At moderate energies, in the limit $\tau_{f} \rightarrow 0$, this treatment reduces to the conventional cascade model [26].

Mean field dynamics is neglected in our consideration. However, we keep the nuclear scalar potential to be defined for the initial state in the local ThomasFermi approximation, changing in time only the potential depth according to the number of knocked-out nucleons. This "frozen mean-field" approximation allows us to take into account nuclear binding and the Pauli principle as well as to estimate the excitation energy of the residual nucleus through excited particle-hole counting. This approximation is good for hadron-nucleus or peripheral nucleus-nucleus collisions where there is no large disturbance of the mean field, but it is getting questionable for violent central collisions of heavy ions. Typical results for the distributions of residual masses $A_{R}$ versus their excitation energies $E_{R}$ in this model are shown in Fig. 2.

It is traditionally assumed that after completing the cascade stage the excited residual nucleus is in an equilibrium state. In general this is not evident. The $\mathrm{RC}$ includes the possibility to describe the attainment of thermodynamic equilibrium in terms of the pre-equilibrium (PE) exciton model [26,30,31]. During this equilibration process some pre-equilibrium particles may be emitted, which will result in the change of characteristics for the thermalized residual nucleus. The influence of this option is discussed in section 4.1. 


\subsection{Statistical Multifragmentation Model}

Within the SMM [27], the probability of a decay from equlibrium into the given channel is proportional to its statistical weight. The calculations are of Monte-Carlo type. The break-up volume $V_{b}$ is one parameter. It is taken as $V_{b}=(1+k) A / \rho_{0}$, where $A$ is the mass number of the fragmenting nucleus, $\rho_{0}$ is the normal nuclear density and $k$ is a free parameter. In Refs. $[12,13,18]$ we have shown that the break up occurs at low density. To reach these densities it is assumed that the system has to expand before the break up. Furthermore, the primary fragments may be excited and their deexcitation is taken into account to get final IMF distributions. Figure 3 shows the IMF multiplicity as a function of the excitation energy calculated for $k=2$ and $k=5$ which corresponds to freeze-out densities of $\approx 1 / 3 \rho_{0}$ and $1 / 6 \rho_{0}$, respectively. The calculations have been performed with the RC+SMM combined model for ${ }^{4} \mathrm{He}+\mathrm{Au}$ collisions at $14.6 \mathrm{GeV}$. The fragment multiplicity rises with excitation energy up to a maximum and then decreases due to vaporization of the overheated system. This so-called "rise and fall" of multifragmentation is well visible in the figure and was first demonstrated experimentally by the ALADIN group for the collisions of ${ }^{197} \mathrm{Au}$ at $600 \mathrm{MeV} /$ nucleon with $\mathrm{Al}$ and $\mathrm{Cu}$ targets [32].

Figure 3 evidences that the choice of the break-up density only slightly influences $\langle M\rangle$. The kinetic energies of fragments are more affected because they are determined mainly by the Coulomb field in the system depending noticeably on its size. The use of a large value of the parameter $(k=5)$ results in the underestimation of the fragment kinetic energies compared to the data [33]. In the calculations presented here we used $k=2$ based on our analysis of the correlation data [18].

\section{Results and Discussion}

\subsection{Fragment multiplicity and excitation energy of the system}

The mean IMF-multiplicities, measured and calculated, are shown in Fig. 4 as a function of the total beam energy for various projectiles. The data exhibit a saturation in $\langle M\rangle$ for incident energies above $\sim 6 \mathrm{GeV}$ in good agreement with findings of previous works $[13,34,35]$.

The dashed line in Fig. 4 is obtained by means of the combined $\mathrm{RC}+\mathrm{SMM}$ model. The calculated mean multiplicities are significantly higher than the measured ones except for the lowest beam energy. This fact indicates that 
the model overestimates the residue excitation energy. The inclusion of preequilibrium (PE) emission after the cascade stage $(\mathrm{RC}+\mathrm{PE}+\mathrm{SMM})$ results in a significant decrease of the excitation energy of the fragmenting target spectator and reduces the mean IMF multiplicity (dotted line in Fig. 4). However, the multiplicity reduction turns out to be too large for $E_{\text {proj }}<8 \mathrm{GeV}$ giving $<$ $M>$ much smaller than the measured ones. One should note that, though the calculated value of $\langle M\rangle$ for the $\mathrm{p}(8.1 \mathrm{GeV})+\mathrm{Au}$ collisions almost coincides with the experiment, the model-predicted fragment kinetic energies in this approach are significantly lower than the measured ones, as shown in [13]. Because the IMF energies are determined essentially by the Coulomb field of the source, the $\mathrm{RC}+\mathrm{PE}+\mathrm{SMM}$ model underestimates the charge $Z$ of the target residue. In addition, at higher ${ }^{4} \mathrm{He}$-beam energies, the drop in excitation energy after the pre-equilibrium emission is not strong enough to get the observed fragment multiplicities. All these facts may testify to the existence of other mechanisms for energy loss before the IMF emission.

From the comparison of the model calculations for the fragment multiplicities with the data we conclude that neither $\mathrm{RC}$ nor $\mathrm{RC}+\mathrm{PE}$ are able to describe the properties of a target spectator over a wide range of projectiles energies.

An example of an empirical approach to this problem is given in paper [36] devoted to an analysis of the experimental data on multifragmentation in the reactions of ${ }^{197} \mathrm{Au}$ on $\mathrm{C}, \mathrm{Al}, \mathrm{Cu}$ and $\mathrm{Pb}$ targets at $E / A=600 \mathrm{MeV}$. The parameterized relations (with 7 parameters) were developed to get the mass and energy distributions of highly excited thermalized nuclear systems formed as the spectator parts of the colliding nuclei. These distributions were used as input for SMM calculations with the parameters adjusted to fit experimental IMF multiplicity distributions and their yields. It should be stressed that the suggested parameterization is specific for the considered reaction.

In our approach we start with the results of the cascade calculation and modify them empirically. As discussed in [37], the excitation energies of the cascade remnants have been reduced by factor $\alpha$ (see below) and we assume that the drop in excitation energy is accompanied by a mass loss. This combination holds both for preequilibrium emission, in the spirit of the exciton model [31], and for particle evaporation during expansion, as considered by the EES model [38]. The excitation energies $E_{R}^{R C}$ of the residual nuclei $A_{R}$ given by the $\mathrm{RC}$ code are reduced by a factor $\alpha$ to get the excitation energy of the multifragmenting state $E_{M F}$, i.e. $E_{M F}=\alpha \times E_{R}^{R C}$. In other words, the drop in the excitation energy is equal to $\Delta E=(1-\alpha) E_{R}^{R C}$. As is known from the cascade calculations, $E_{R}^{R C}$ is proportional to the nucleon loss during the cascade $\Delta A^{R C}$, so $\Delta E=(1-\alpha) \varepsilon_{1} \Delta A^{R C}$, where $\varepsilon_{1}$ is a mean excitation energy per ejected cascade nucleon. The loss in mass $\Delta A$ corresponding to this drop in excitation energy is $\Delta A=\Delta E / \varepsilon_{2}$, where $\varepsilon_{2}$ is the mean energy removed by a nucleon. Assuming $\varepsilon_{2} \approx \varepsilon_{1}$ one gets $\Delta A=(1-\alpha) \Delta A^{R C}$. We denote this 
empirical combined model as $\mathrm{RC}+\alpha+\mathrm{SMM}$.

In the earlier paper [13] for $\mathrm{p}+\mathrm{Au}$ collisions, a simple relation to determine $\alpha$ could be applied

$$
\alpha=\frac{<M_{\text {exper }}>}{<M_{I N C+S M M}>}
$$

because the range of the excitation energies corresponded to the rising part of the energy dependence of $\langle M\rangle$ shown in Fig. 3. However, due to the rise-and-fall effect in $\langle M\rangle$, this relation fails for heavier projectiles. For these systems the values of $\alpha$ are empirically adjusted to reproduce the measured mean IMF multiplicities. The charge, mass and energy characteristics of fragmenting nuclei resulting from this fitting procedure are presented in Table 1 for various colliding systems. The corresponding values for the $\mathrm{p}+\mathrm{Au}$ case differ slightly from those given in Ref. [13] because a new cascade code is used here. The values of the parameter $\alpha$ can be obtained from Table 1 by calculating the ratio $E_{R}(\mathrm{RC}+\alpha+\mathrm{SMM}) / E_{R}(\mathrm{RC}+\mathrm{SMM})$ which gives $0.93,0.76$ and 0.53 (for $\mathrm{p}+\mathrm{Au}$ ), 0.49 and 0.25 (for $\mathrm{He}+\mathrm{Au}$ ), 0.22 (for $\mathrm{C}+\mathrm{Au}$ ), respectively.

As follows from the given values of the parameter $\alpha$, a rather large decrease of the residual excitation energy is required to reproduce the observed saturation effect in $\langle M\rangle$ which is caused mainly by a saturation in $E_{M F}$. This is illustrated in Fig. 5 which shows the population of events in the $M-$ versus- $E_{M F} / A_{M F}$ plane calculated in both the RC+SMM (left panel) and $\mathrm{RC}+\alpha+\mathrm{SMM}$ (right panel) scenarios. According to the first approach the excitation energy distribution is rather wide and populates states along both the rising and the falling parts of the multiplicity curve. In the $\mathrm{RC}+\alpha+\mathrm{SMM}$ scenario the events are mainly situated in the rising part hardly approaching the region of maximal values of the IMF multiplicity.

The calculated mean residual excitation energies and mean mass numbers are presented in Fig. 6. The total excitation energy of the fragmenting nucleus $E_{M F}$ changes slightly with an increase of the incident energy. At the same time, the excitation energy per nucleon goes up while the residual mass decreases, keeping the mean IMF multiplicity almost constant. Note that the energies given in Fig. 6 and Table 1 are thermal ones by definition. A possible nonthermal contribution will be discussed at the end of this section along with Fig. 15.

It is of interest to compare the extracted masses and excitation energies of fragmenting nuclei to those obtained by the EOS collaboration for $\mathrm{Au}(1$ $\mathrm{GeV} /$ nucleon) $+\mathrm{C}$ collisions (in inverse kinematics) [39]. In that paper the mass and energy balance relations were applied using of the measured kinetic energies of all outgoing charged particles after separating from the prompt stage of the reaction. The neutron contribution was taken into account on the basis of cascade and statistical model simulations. The inclusive data were 


\begin{tabular}{|c|c|c|c|c|c|c|c|c|c|c|}
\hline \multirow{2}{*}{$\begin{array}{c}E_{\text {inc }} \\
(\mathrm{GeV})\end{array}$} & \multirow[t]{2}{*}{ Proj } & \multirow{2}{*}{$\begin{array}{l}\text { Exper. } \\
M_{I M F}\end{array}$} & \multicolumn{7}{|c|}{ Calculations } & \multirow[t]{2}{*}{ Model } \\
\hline & & & $M_{I M F}$ & $Z_{R}$ & $A_{R}$ & $Z_{M F}$ & $A_{M F}$ & $E_{R}$ & $E_{M F}$ & \\
\hline \multirow{3}{*}{2.16} & \multirow{3}{*}{$\mathrm{p}$} & \multirow{3}{*}{$1.7 \pm 0.2$} & 1.82 & 77 & 189 & 76 & 185 & 310 & 589 & $\mathrm{RC}+\mathrm{SMM}$ \\
\hline & & & 1.02 & 72 & 176 & 62 & 145 & 119 & 266 & $\mathrm{RC}+\mathrm{PE}+\mathrm{SMM}$ \\
\hline & & & 1.69 & 77 & 188 & 75 & 183 & 288 & 564 & $\mathrm{RC}+\alpha+\mathrm{SMM}$ \\
\hline \multirow{3}{*}{3.6} & \multirow{3}{*}{$\mathrm{p}$} & \multirow{3}{*}{$1.9 \pm 0.2$} & 2.52 & 76 & 187 & 74 & 181 & 371 & 676 & $\mathrm{RC}+\mathrm{SMM}$ \\
\hline & & & 1.34 & 70 & 171 & 55 & 134 & 148 & 385 & $\mathrm{RC}+\mathrm{PE}+\mathrm{SMM}$ \\
\hline & & & 1.89 & 75 & 184 & 73 & 175 & 282 & 568 & $\mathrm{RC}+\alpha+\mathrm{SMM}$ \\
\hline \multirow{3}{*}{8.1} & \multirow{3}{*}{$\mathrm{p}$} & \multirow{3}{*}{$2.1 \pm 0.2$} & 3.58 & 75 & 183 & 73 & 175 & 488 & 808 & $\mathrm{RC}+\mathrm{SMM}$ \\
\hline & & & 1.85 & 68 & 167 & 53 & 128 & 177 & 462 & $\mathrm{RC}+\mathrm{PE}+\mathrm{SMM}$ \\
\hline & & & 2.0 & 72 & 176 & 67 & 158 & 259 & 529 & $\mathrm{RC}+\alpha+\mathrm{SMM}$ \\
\hline \multirow{3}{*}{4.0} & \multirow{3}{*}{${ }^{4} \mathrm{He}$} & \multirow{3}{*}{$1.7 \pm 0.2$} & 3.89 & 75 & 184 & 73 & 177 & 484 & 836 & $\mathrm{RC}+\mathrm{SMM}$ \\
\hline & & & 1.56 & 68 & 167 & 54 & 130 & 176 & 428 & $\mathrm{RC}+\mathrm{PE}+\mathrm{SMM}$ \\
\hline & & & 1.77 & 73 & 177 & 69 & 161 & 238 & 502 & $\mathrm{RC}+\alpha+\mathrm{SMM}$ \\
\hline \multirow{3}{*}{14.6} & \multirow{3}{*}{${ }^{4} \mathrm{He}$} & \multirow{3}{*}{$2.2 \pm 0.2$} & 4.47 & 71 & 173 & 66 & 159 & 723 & 1132 & $\mathrm{RC}+\mathrm{SMM}$ \\
\hline & & & 3.06 & 63 & 153 & 48 & 116 & 377 & 824 & $\mathrm{RC}+\mathrm{PE}+\mathrm{SMM}$ \\
\hline & & & 2.19 & 64 & 154 & 48 & 103 & 183 & 404 & $\mathrm{RC}+\alpha+\mathrm{SMM}$ \\
\hline \multirow{3}{*}{22.4} & \multirow{3}{*}{${ }^{12} \mathrm{C}$} & \multirow{3}{*}{$2.2 \pm 0.3$} & 4.04 & 67 & 163 & 64 & 153 & 924 & 1216 & $\mathrm{RC}+\mathrm{SMM}$ \\
\hline & & & 2.85 & 60 & 146 & 47 & 113 & 638 & 1026 & $\mathrm{RC}+\mathrm{PE}+\mathrm{SMM}$ \\
\hline & & & 2.17 & 59 & 139 & 41 & 86 & 207 & 415 & $\mathrm{RC}+\alpha+\mathrm{SMM}$ \\
\hline
\end{tabular}

Table 1

The calculated properties of nuclear remnants from proj $+\mathrm{Au}$ collisions. The $M_{I M F}$ is the mean number of IMF's for events with at least one IMF and $Z_{R}, A_{R}$, $E_{R}$ are the mean charge, mass number and excitation energy (in $\mathrm{MeV}$ ), respectively, averaged over all inelastic collisions, while similar quantities $Z_{M F}, A_{M F}, E_{M F}$ are averaged only over residues decaying by IMF emission. The errors in $A_{M F}$ range from $3 \%$ to $14 \%$ and in $E_{M F}$ from $10 \%$ to $20 \%$, the lower value refers to p $(8.1 \mathrm{GeV})$ and the highest to ${ }^{12} \mathrm{C}(22.4 \cdot \mathrm{GeV})$.

not presented there and only the values, corresponding to our mean IMF multiplicity were used for the comparison. Our value of $E_{M F} / A_{M F}$ is close to that from Ref. [39] if a collective energy is added (see later). As to the mean mass $A_{M F}$, the value obtained in the present work $(\approx 90)$ is remarkably lower, because of the larger mass loss induced by a projectile with twice the energy.

Some examples of the excitation energy distributions are displayed in Fig. 7. The IMF emission takes place on the tail of the distributions (hatched area), 
therefore the mean excitation of the fragmenting nuclei is much larger than that averaged over all the target spectators.

\subsection{Angular distributions}

Now let us consider the thermalization of the system at break-up. To check whether this state of the emitting system is close to thermal equilibrium, the plot of the fragment invariant probability distribution in terms of the longitudinal-versus-transversal velocity components is presented in Fig. 8 for the ${ }^{4} \mathrm{He}+\mathrm{Au}$ and $\mathrm{C}+\mathrm{Au}$ collisions. Circles connect experimental points of equal invariant cross sections for emitted carbon fragments in the energy range above the spectral peak. This demonstrates an isotropic emission in the frame of a moving source and indicates that the fragment emission proceeds from a thermalized state with the center positions of the circles determining the longitudinal source velocity, $\beta_{\text {source }}$. Their mean values are close to estimates with the $\mathrm{RC}+\alpha+\mathrm{SMM}$ model for all cases except ${ }^{4} \mathrm{He}+\mathrm{Au}$ at $4 \mathrm{GeV}$, where the calculations underestimate the source velocity by almost a factor of two. The calculated mean $\beta_{\text {source }}$ are equal to $0.76 \cdot 10^{-2}, 1 \cdot 10^{-2}, 1.36 \cdot 10^{-2}$ and $1.7 \cdot 10^{-2}$ for $\mathrm{p}(8.1 \mathrm{GeV})+\mathrm{Au},{ }^{4} \mathrm{He}(4 \mathrm{GeV}),{ }^{4} \mathrm{He}(14.6 \mathrm{GeV})+\mathrm{Au}$ and $\mathrm{C}(22.4 \mathrm{GeV})+\mathrm{Au}$ collisions, respectively.

The fragment angular distribution in the laboratory system exhibits a forward peak caused by the source motion as exemplified in Fig. 9 for carbon fragments. The data are well reproduced by the model calculations except those for the helium beam at the lowest energy. The measured distribution here is more forward peaked, which may be considered as an indication that the momentum transfer is larger than predicted. This observation may indicate a stronger stopping than predicted for this case.

\subsection{Charge distributions}

The charge distributions of IMF's are shown in Fig. 10. The calculations for the $\mathrm{RC}+\alpha+\mathrm{SMM}$ scenario agree nicely with the data (left side of Fig. 10). The general trend of the IMF charge (or mass) distributions is well described by a power law $Y(Z) \sim Z^{-\tau}$. The obtained values of the exponent are $\tau=$ $2.17 \pm 0.08,1.90 \pm 0.06$ and $1.88 \pm 0.06$ for proton beam of $2.1 \mathrm{GeV}, 3.6 \mathrm{GeV}$ and $8.1 \mathrm{GeV}, \tau=1.93 \pm 0.06$ and $2.0 \pm 0.1$ for helium beam of $4 \mathrm{GeV}$ and 14.6 $\mathrm{GeV}$ and finally $\tau=2.1 \pm 0.1$ for carbon projectiles (Fig. 10, right panel).

In earlier papers on multifragmentation $[5,9]$ this power-law behavior of the IMF yield was interpreted as an indication of the proximity of the decaying state to the critical point for the liquid-gas phase transition in nuclear mat- 
ter. This was stimulated by the application of the classical Fisher's droplet model [40], which predicted a pure power-law droplet-size distribution with $\tau=2-3$ at the critical point. According to Ref. [41] the fragmenting system is not close to the critical point.

The power law is well explained at temperatures far below the critical point. As seen in Fig. 10, the pure thermodynamical SMM predicts that the IMF charge distribution is very close to a power law at freeze-out temperatures of 5-7 MeV, while the critical temperature $T_{c}$ (at which the surface tension vanishes) is assumed to be $18 \mathrm{MeV}$. In Ref. [42], it was also shown that several results concerning the fragment-size distribution (a power-law behaviour) can be rendered well by the use of the kinetic model of condensation beyond the vicinity of the liquid-gas critical point. The critical temperature and density of nuclei were calculated in Ref. [6] using a Skyrme effective interaction and finite-temperature Hartree-Fock theory. The values of $T_{c}$ were found to be in the range (8.1-20.5) MeV depending on the Skyrme-interaction parameters chosen.

Thermal multifragmentation is consistent with a first-order phase transition of nuclear matter characterized by the liquid-gas type phase instability. Indeed, it is consistent with experiments that fragmentation takes place after expansion driven by thermal pressure [12-14], and that the decomposition time is short (less than $100 \mathrm{fm} / \mathrm{c}$ ) [17-19]. In fact, the final state of this transition looks like a nuclear fog [43] : the liquid drops of IMF's surrounded by a gas of nucleons and light clusters, $d, t$ and $\alpha$-particles. This interpretation is in the line of the SMM [44]. Recently, several theoretical and experimental papers have been published on that topic (see for example [50]). An interesting aspect of this concept is the isospin fractionation as was demonstrated in Ref. [45].

\subsection{Energy spectra of fragments}

In general, the kinetic energy of fragments is determined by the sum of four terms: thermal motion, Coulomb repulsion, rotation and collective expansion energies of the system at freeze out: $E=E_{t h}+E_{C}+E_{\text {rot }}+E_{\text {flow }}$. The additivity

of the first three terms is quite obvious. For the last term, its independence of the others may be considered only approximately when the evolution of the system after the freeze-out point is driven only by the Coulomb force. The Coulomb term is significantly larger than the thermal one. It was shown in Ref. [18] that the Coulomb part of the mean energy of the carbon fragment is three times larger than thermal energy. These calculations were performed within the $\mathrm{RC}+\mathrm{SMM}$ scenario with the volume emission of fragments from a diluted system. 
The contribution of the collective flow for the $\mathrm{p}+\mathrm{Au}$ collisions at $8.1 \mathrm{GeV}$ incident energy was estimated in Ref. [13]. This was done by comparing the measured IMF spectra with the calculated ones in the framework of the SMM which includes no flow. This analysis did not reveal any significant enhancement in the measured energy spectra restricting the mean flow velocity $v_{\text {flow }}$ to less than $0.02 c$. For the case of heavy ion collisions, collective flow has been observed and it is the most pronounced in central $\mathrm{Au}+\mathrm{Au}$ collisions [46]. In this respect it would be quite interesting to analyse the fragment spectra from $\mathrm{He}+\mathrm{Au}$ and $\mathrm{C}+\mathrm{Au}$ collisions looking for a possible manifestation of collective flow. Indeed, a comparison of the energy spectra of carbon fragments emitted in $\mathrm{p}(8.1 \mathrm{GeV})+\mathrm{Au}$ and $\alpha(14.6 \mathrm{GeV})+\mathrm{Au}$ shows a drastic difference as demonstrated in Fig. 11. A more general comparison of the carbon spectra for proton-, helium- and carbon-induced collisions on the Au target are presented in Fig. 12. The calculated carbon spectrum for $\mathrm{p}+\mathrm{Au}$ collisions (at $8.1 \mathrm{GeV}$ ) is consistent with the measured one. A similar situation occurs with ${ }^{4} \mathrm{He}+\mathrm{Au}$ collisions at $4 \mathrm{GeV}$, but not with ${ }^{4} \mathrm{He}(14.6 \mathrm{GeV})+\mathrm{Au}$ and ${ }^{12} \mathrm{C}(22.4 \mathrm{GeV})+\mathrm{Au}$ interactions: the measured spectra are harder than the calculated ones.

The measured mean kinetic energies per nucleon $<E>/ A_{I M F}$ are given in the upper part of Fig. 13, only statistical errors are shown. Systematic errors $(\sim 5 \%)$ are the same for all the cases. They can be neglected when comparing data obtained for different collision systems. There is a remarkable enhancement in the reduced kinetic energy for light fragments from $\mathrm{He}(14.6$ $\mathrm{GeV})+\mathrm{Au}$ and $\mathrm{C}(22.4 \mathrm{GeV})+\mathrm{Au}$ collisions as compared to the $\mathrm{p}(8.1 \mathrm{GeV})+\mathrm{Au}$ case. The calculated values of the mean fragment energies (shown by lines) are obtained with the $\mathrm{RC}+\alpha+\mathrm{SMM}$ model by multibody Coulomb trajectory calculations on an event-by-event basis. In the initial state all charged particles are assumed to have a thermal velocity only. The measured energies are close to the calculated ones for $\mathrm{p}+\mathrm{Au}$ collisions in the range of the fragment charges between 4 and 9. However, for the ${ }^{4} \mathrm{He}+\mathrm{Au}$ and ${ }^{12} \mathrm{C}+\mathrm{Au}$ interactions the experimental data exceed the calculated values.

The observed deviation is not caused by any methodical distortion of the spectral shape for heavier beams, e.g. by the pile-up effect. Its magnitude is determined by the counting rates, which are comparable for $\mathrm{p}$ and He beams and are smaller for $\mathrm{C}+\mathrm{Au}$ collisions.

This deviation cannot be attributed to an angular momentum effect. This has been estimated in Ref. [21] to be $\left\langle E_{\text {rot }}>/ A_{I M F} \approx 0.04 \mathrm{MeV} /\right.$ nucleon, which is an order of magnitude smaller than the energy enhancement for light fragments. We suggest that this enhancement is caused by the expansion of the system, which is assumed to be radial as the velocity plot (Fig. 8) does not show any significant deviation from circular symmetry.

An estimate of the fragment flow energy may be obtained as the difference 
between the measured IMF energies and those calculated without taking into account any flow in the system. This difference for $\mathrm{C}+\mathrm{Au}$ collisions is shown in the middle part of Fig. 13. The error bars include both statistical and systematic contributions. In an attempt to describe the data we modified the SMM code in the $\mathrm{RC}+\alpha+\mathrm{SMM}$ concept by the inclusion of a radial velocity boost for each particle at freeze out. In other words, a radial expansion velocity was superimposed on the thermal motion in the calculation of the multibody Coulomb trajectories. A self-similar radial expansion is assumed, where the local flow velocity depends linearly on the distance of the particle from the centre of mass. The expansion velocity of a particle with charge $Z$ located at radius $R_{Z}$ is given by

$$
\vec{v}_{\text {flow }}(Z)=v_{\text {flow }}^{0} \cdot \frac{\vec{R}_{Z}}{R_{\text {sys }}}
$$

where $v_{\text {flow }}^{0}$ is the radial velocity at the surface of the system. Note, that in this case the density distribution is changing in a dynamic evolution by a self-similar way being a function of the scaled radius $R_{Z} / R_{\text {sys }}$. The use of the linear profile for the radial velocity is motivated by hydrodynamic model calculations of an expanding hot nuclear system (see for example Ref. [47]). The value of $v_{\text {flow }}^{0}$ has been adjusted to describe the mean kinetic energy measured for carbon fragments. Figure 14 shows the comparison of the measured and calculated energy spectra of carbon fragments assuming $v_{\text {flow }}^{0}=0.1 c$. The agreement is very good. The calculation without flow deviate strongly.

There is a longstanding problem of the qualitative difference between the chemical or thermal equilibrium temperatures and the kinetic or, so called, slope temperatures. The last discussion on that point can be found in [51]. The mean equilibrium temperature obtained in our calculations is $6.9 \mathrm{MeV}$. At the same time, the slope temperature (inverse slope parameter) extracted from the spectra for pure thermal decay is $T_{s}=14.5 \mathrm{MeV}$ (dashed curve). That is the mutual result of the thermal motion, Coulomb repulsion during the volume disintegration and the secondary decay of the excited fragments. Introducing rather modest radial flow (with $v_{\text {flow }}^{0}=0.1 c$ ) results in increase of the slope temperature up to $T_{s}=24 \mathrm{MeV}$.

Lets return to Fig. 13 middle. Dashed line presents the difference of calculated fragment energies obtained for $v_{\text {flow }}^{0}=0.1 c$ and $v_{\text {flow }}^{0}=0$.

The data deviate significantly from the calculated values for $\mathrm{Li}$ and Be. This may be caused in part by the contribution of particle emission during the early stage of expansion from the hotter and denser system. It is supported by the fact that the extra energy of Li fragments with respect to the calculated value is clearly seen in Fig. 13 even for proton-induced fragmentation, where no significant flow is expected. This peculiarity of light fragments has been 
noted already by the ISIS group for ${ }^{3} \mathrm{He}+\mathrm{Au}$ collisions at $4.8 \mathrm{GeV}[24]$.

For fragments heavier than carbon, the calculated curve in the middle part of Fig. 13 is above the data and decreases only slightly with increasing fragment charge. Such a behaviour is expected. The mean fragment flow energy is proportional to $\left\langle R_{Z}^{2}\right\rangle$. This value is only slightly changing with fragment charge in the SMM code due to the assumed equal probability for fragments of a given charge to be formed at any point in the available break-up volume. This assumption is a consequense of the simplification of the model to consider the system as uniform with $\rho(r)=$ const for $r \leq R_{\text {sys }}$. The discrepancy between the data and the calculations in Fig. 13 indicates that a uniform density distribution is not fulfilled. The dense interior of the expanded nucleus is favored for the appearance of larger IMF's, if fragments are formed via the density fluctuations. This observation is in accordance also with the analysis of the mean IMF energies performed in Ref. [9,13] for proton-induced fragmentation. It is seen also in Fig. 13, that for $\mathrm{p}+\mathrm{Au}$ collisions the measured energies are below the theoretical curve for fragments heavier than Ne. This may be explained by the preferential location of the heavier fragments in the interior region of the freeze out volume, where the Coulomb field is reduced. The deviation of data from the calculations becomes less, but still remains, if one assumes a quadratic radial profile for the expansion velocity.

For the estimation of the mean flow velocities of fragments, the difference between the measured IMF energies and calculated ones (no flow) has been used as discussed already in Ref. [21]. The results are presented in the lower part of Fig. 13. The values for Li and Be are considered as upper limits because of the possible contribution of preequilibrium emission. The corresponding values of $\left\langle R_{Z}>/ R_{\text {sys }}\right.$, obtained under the assumption of a linear radial profile for the expansion velocity, can be read on the right-hand scale of the figure. Again the mean radial coordinate for the carbon fragment is chosen to coincide with the calculated one. The dashed line shows the mean radial coordinates of fragments according to the SMM code. As it has been noted above, the calculated values of $\left\langle R_{Z}>/ R_{\text {sys }}\right.$ are only slightly decreasing with $Z$ in contrast with the data.

The flow velocity on the system surface obtained in this analysis is close to the value of the maximum expansion velocity (0.08c) calculated with EES for ${ }^{12} \mathrm{C}+\mathrm{Au}$ collisions [52].

Effects of the radial collective energy for $1 \mathrm{GeV} /$ nucleon $\mathrm{Au}+\mathrm{C}$ collisions (in inverse kinematics) were considered in [48] by analyzing the transverse kinetic energies $E_{t}$ of fragments with $Z=2-7$. This was done for two chargedparticle multiplicity bins, corresponding to peripheral (M1) and central (M3) collisions. The Berlin statistical model code [49] was used with inclusion of a radial velocity chosen properly to account for the experimental values of $<$ 
$E_{t}>$. In the case of peripheral collisions the obtained expansion velocities are close to those extracted here, but the corresponding mean IMF multiplicities (in our definition) are lower than 1.5. For central collisions $\left(<M_{I M F}>\simeq\right.$ 4) the expansion velocities are $\sim 1.5$ times higher. It would be desirable to compare our data with those for the intermediate case (bin M2), which are unfortunately not available. Making an interpolation, one may see that our analysis gives slightly lower values of $v_{\text {flow }}(Z)$ as compared to Ref. [48]. This may be caused by the fact that the Berlin statistical model underestimates the Coulomb part of the fragment kinetic energy (see [33]) as the freeze-out density used is much smaller $\left(\rho_{f}=1 / 6 \rho_{0}\right)$.

The total expansion energy can be estimated by integrating the nucleon flow energy (taken according to Eq. (2)) over the available volume at freeze out. For a uniform system one gets:

$$
E_{\text {flow }}^{\text {tot }}=\frac{3}{10} A \cdot m_{N}\left(v_{\text {flow }}^{0}\right)^{2}\left(1-r_{N} / R_{\text {sys }}\right)^{5}
$$

where $m_{N}, r_{N}$ are the nucleon mass and radius. For ${ }^{12} \mathrm{C}+\mathrm{Au}$ collisions it gives $E_{\text {flow }}^{\text {tot }} \simeq 115 \mathrm{MeV}$, corresponding to the flow velocity on the surface equal $0.1 c$. Similar results are obtained for ${ }^{4} \mathrm{He}(14.6 \mathrm{GeV})+\mathrm{Au}$ collisions.

The excitation energy of the fragmenting systems consists of a thermal part $E_{M F}$ given in Table 1 and, in some cases, an additional dynamical part $E_{\text {flow }}^{\text {tot }}$. These findings are reflected in Fig. 15 where the total excitation energy $E_{M F}^{*} / A_{M F}$ is shown as a function of the incident energy. The full symbols correspond to the thermal part of the excitation energies. These values exhibit a rather weak rise with increasing incident energy. Adding the flow energy (grey area, open symbols) yields a stronger increase. The onset of the collective flow takes place at excitation energies around $4 \mathrm{MeV} /$ nucleon, which is in good agreement with the results of [15]. In contrast to Ref. [16], we do not find arguments that this onset should be related to a transition from a surface to a bulk emission.

\section{Conclusions}

The emission of intermediate mass fragments has been studied in the reactions $\mathrm{p}(2.1,3.6$ and $8.1 \mathrm{GeV})+\mathrm{Au},{ }^{4} \mathrm{He}(4$ and $14.6 \mathrm{GeV})+\mathrm{Au}$ and ${ }^{12} \mathrm{C}(22.4$ $\mathrm{GeV})+\mathrm{Au}$. The measured IMF multiplicities (for events with at least one IMF) saturate at a value of $2.2 \pm 0.2$ for incident energies above $6 \mathrm{GeV}$. This saturation in IMF multiplicity seems to correspond to total excitation energies around $3-4 \mathrm{MeV} /$ nucleon. The angular distributions of the IMF's are slightly forward peaked; the yield distributions of parallel versus perpendic- 
ular velocities exhibit circular symmetry. These results show that the IMF's are emitted from a source that moves with rather low velocity $(0.01-0.02) c$ and support the interpretation of "thermal multifragmentation", a break-up of a diluted system.

Model calculations for the IMF multiplicities using a two-stage concept, a cascade followed by SMM (statistical multifragmentation model), fail to describe the measured values. Taking into account pre-equilibrium particle emission before attainment of thermal equilibrium in the system decreases the number of IMF's, but this is still not sufficient for describing the observed multiplicity saturation. Only if one applies an empirical modification of the calculated excitation energies $E_{R}$ and residual masses $A_{R}$ after the cascade used as input for the SMM calculations, the observed saturation of the IMF multiplicity can be reproduced. This study shows that an intermediate step is needed which likely reflects an expansion before the freeze-out density is reached. This picture resembles the dynamics of the EES model which has an expansion phase before the bulk of the IMF's are emitted. This expansion phase might gradually happen faster for higher incident energies and most likely for heavier projectiles as indicated in the measured energy spectra.

The energy spectra of the IMF's turn out to be sensitive observables. In p+Au collisions, the energy spectra are well described by the empirically modified cascade-SMM calculations. However, for ${ }^{4} \mathrm{He}$ and ${ }^{12} \mathrm{C}$ induced reactions the spectra exhibit stronger high-energy tails which cannot be reproduced by the calculations. This effect cannot be accounted for by any variation of the residual masses. We attribute this observation to the persistence of collective flow in the system at freeze-out. Assuming a linear radial profile for the flow velocity, its value at the surface is estimated to be around $0.1 c$ both for ${ }^{4} \mathrm{He}$ and ${ }^{12} \mathrm{C}$ induced reactions. However, a detailed inspection of the variation of the kinetic energies of the fragments reveals that the flow velocities seem to vary with the fragment charge. This is in contrast to model expectations which assume equal probability for fragments of a given charge to be formed at any point in the break-up volume. This discrepancy indicates that heavier fragments are formed more in the interior of the system, possibly due to a density gradient.

This study of multifragmentation using a range of projectiles from protons to light nuclei provides new information on several aspects of multifragmentation. It demonstrates a transition from a pure "thermal decay" (for $\mathrm{p}+\mathrm{Au}$ Collisions) to a disintegration characterized by a collective flow (for heavier projectiles). Nevertheless, the decay mechanism looks like a thermal multifragmentation, as the partition of the system is governed by the nuclear temperature. In all cases the IMF charge distributions are well described by statistical multifragmentation models without flow. This transition occurs at incident energies around $6 \mathrm{GeV}$ using light projectiles. Our study puts into question the 
usual two-stage concept for describing multifragmentation. Furthermore, a realistic, non-uniform density distribution might be needed to properly describe the phase-space occupancy at freeze out.

The authors are thankful to Profs. A. Hrynkiewicz, A.N. Sissakian, A.I. Malakhov, N.A. Russakovich and S.T. Belyaev for support. The research was supported in part by Grant No 00-02-16608 from Russian Foundation for Basic Research, by Grant No 2P03 12615 from the Polish State Committe for Scientific Research and Grant of the Polish Plenipotentiary in JINR, by Grant No 94-2249 from INTAS, by Contract No 06DA819 with Bundesministerium

für Forschung und Technologie, by Grant PST.CLG.976861 from NATO, and by US National Science Foundation.

\section{References}

[1] E. Schopper, Naturwissenschafter 25 (1937) 557.

[2] I.I. Gurevich et al., Dokl. Akad. Nauk SSSR 18 (1938) 169.

[3] O.V. Lozhkin, N.A. Perfilov, Zh. Eksp. i Teor. Fiz. 31 (1956) 913; N.A. Perfilov et al., Usp. Fiz. Nauk, 70 (1960) 3, (Sov. Phys. Usp. 3,1 (1960)).

[4] B. Jacobsson et al., Z. Phys. A307 (1982) 293.

[5] P.J. Siemens, Nature 305 (1983) 410.

[6] H.R. Jaqaman A.Z. Mekjian, and L. Zamick, Phys. Rev. C27 (1983) 2782; C29 (1984) 2067.

[7] M.W. Curtin et al., Phys. Lett. B123 (1983) 289.

[8] J.H. Rose et al., Phys. Rev. Lett. 53 (1984) 289.

[9] A.S. Hirsch et al., Phys. Rev. C29 (1984) 508.

[10] "Multifragmentation", Proc. of Int. Workshop XXVII on Gross Properties of Nuclei and Nuclear Excitations, Hirschegg, Austria, Jan. 17-23, 1999, Edited by H.Feldmeier, J.Knoll, W.Nörenberg, J.Wambach, GSI, Darmstadt, 1999.

[11] S.J. Yennello et al., Phys. Rev. Lett. 67 (1991) 671.

[12] Bao-An Li, D.H.E. Gross, V. Lips, H. Oeschler, Phys. Lett. B335 (1994) 1.

[13] S.P. Avdeyev et al., Eur. Phys. J. A3 (1998) 75.

[14] K. Kwiatkowski et al., Phys. Rev. Lett., 74 (1995) 3756.

[15] L. Beaulieu et al., Phys. Lett. B463 (1999) 159.

[16] T. Lefort et al., Phys. Rev. C62 (2000) 031604. 
[17] V. Lips et al., Phys. Lett. B338 (1994) 141.

[18] S.Y. Shmakov et al., Yad. Fiz. 58 (1995) 1735; (Phys. of Atomic Nucl. 58 (1995) 1635; V.K.Rodionov et al., Nucl. Phys. A700 (2002) 457.

[19] G. Wang et al., Phys. Rev. C53 (1996) 1811.; G. Wang et al. Phys. Rev. C57 (1998) R2786.

[20] O. Shapiro, D.H.E. Gross, Nucl. Phys. A573 (1994) 143.

[21] S.P. Avdeyev et al., Phys. Lett. B503 (2001) 256.

[22] S.P. Avdeyev et al., Nucl. Instrum. Meth. A332 (1993) 149.

[23] S.P. Avdeyev et al., Pribory i Tekhnika Eksper. 39 (1996) 7; (Instr. Exp. Techn. 39 (1996) 153).

[24] E.R. Foxford et al., Phys. Rev. C54 (1996) 749.

[25] A.S. Botvina, A.S. Iljinov, I.N. Mishustin, Nucl. Phys. A507 (1990) 649; A.S. Botvina et al., Phys. of Atomic Nuclei 57 (1994) 628.

[26] V.D. Toneev, K.K. Gudima, Nucl. Phys. A400 (1983) 173c.

[27] J. Bondorf et al., Phys. Rep. 257 (1995) 133; Nucl. Phys. A444 (1985) 476.

[28] V.D. Toneev et al., Nucl. Phys. A519 (1990) 463c.

[29] N.S. Amelin et al., Yad. Fiz. 52 (1990) 272 (Translated as Sov. Journ. of Nuclear Phys. 52 (1990) 172).

[30] K.K. Gudima et al., Yad. Fiz. 21 (1975) 602 (Translated as Sov. Journ. of Nuclear Phys. 21 (1975) 502).

[31] M. Blann, Ann. Rev. Nucl. Sci. 25 (1975) 123.

[32] C.A. Ogilvie et al., Phys. Rev. Lett. 67 (1991) 1214.

[33] H. Oeschler et al., Particles and Nuclei, Letters No 2 [99] (2000) 70.

[34] K.B. Morlet et al., Phys. Lett. B355 (1995) 52.

[35] N.T. Porile et al., Phys. Rev. C39 (1989) 1914.

[36] A.S. Botvina et al., Nucl. Phys. A584 (1995)737.

[37] S.P. Avdeyev et al., JINR Rapid Communications 2[82] (1997) 71.

[38] W.A. Friedman, Phys. Rev. C42 (1990) 667.

[39] J.A. Hauger et al., Phys. Rev. Lett. 77 (1996) 235; R.P. Scharenberg et al., Phys. Rev. C64 (2001) 054602.

[40] M.E. Fischer, Physics 3 (1967) 255.

[41] V.A. Karnaukhov, Phys. of Atomic Nuclei 60 (1997) 1780. 
[42] J. Schmelzer, G. Röpke, F.-P. Ludwig, Phys. Rev. C55 (1997) 1917.

[43] P.J. Siemens, Nucl. Phys. A428 (1984) 189c.

[44] I.N. Mishustin, Nucl. Phys. A447 (1985) 67c.

[45] H.S. Xu et al., Phys. Rev. Lett. 85 (2000) 716; A.S. Botvina and I.M. Mishustin, Phys. Rev. C63 (2001) 061601R.

[46] W. Reisdorf et al., Nucl. Phys. A612(1997)493; G.D. Kunde et al., Phys. Rev. Lett. 74 (1995) 38.

[47] J.P. Bondorf et al., Nucl. Phys. A296 (1978) 320.

[48] J. Lauret et al., Phys. Rev. C57 (1998) R1051.

[49] D.H.E. Gross, Rep. Progr. Phys. 53 (1990) 605.

[50] S.J. Lee and A.Z. Mekjian, Phys. Rev. C56(1997)2621; V. Baran, M. Colona, M. Di Toro, A.B. Larionov, Nucl. Phys. A632(1998)287; M. D'Agostino et al., Nucl. Phys. A650(1999)329; B. Borderie et al., Phys. Rev. Lett. 86(2001)3252; O. Lopez, Nucl. Phys. A685(2001)246c.

[51] T. Odeh et al., Phys. Rev. Lett. 84(2000)4557.

[52] W.A. Friedman, private communication (2000). Phys. Rev. C27(1983)2782. 

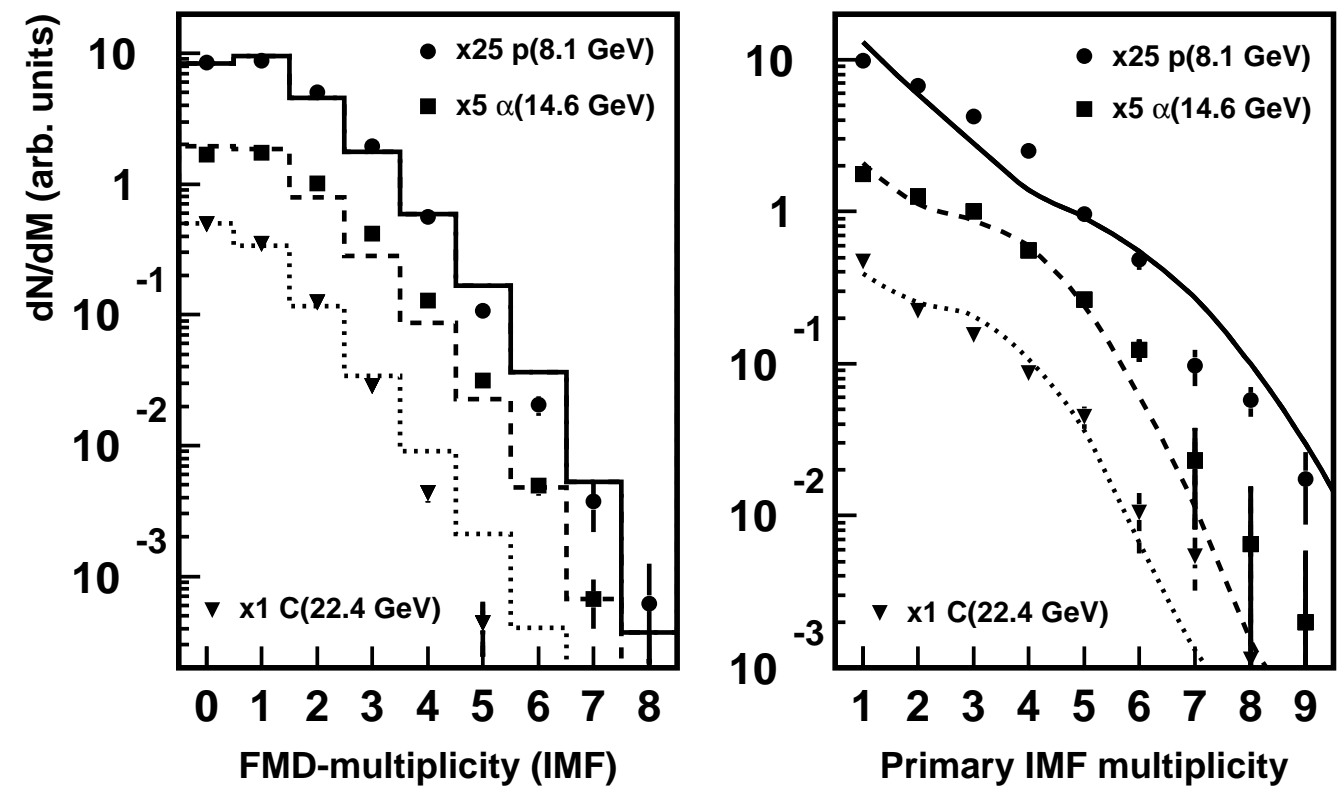

Fig. 1. a) Measured IMF-multiplicity distributions associated with a trigger fragment and fits with a Fermi functions (histograms) for $\mathrm{p}+\mathrm{Au}$ collisions at $8.1 \mathrm{GeV}$ (circles, solid line), ${ }^{4} \mathrm{He}+\mathrm{Au}$ at $14.6 \mathrm{GeV}$ (squares, dashed line), and ${ }^{12} \mathrm{C}+\mathrm{Au}$ at $22.4 \mathrm{GeV}$ (triangles, dotted line). b) Symbols represent directly reconstructed primary IMF distributions. The smooth lines are calculated with the $\mathrm{RC}+\alpha+\mathrm{SMM}$ model. 


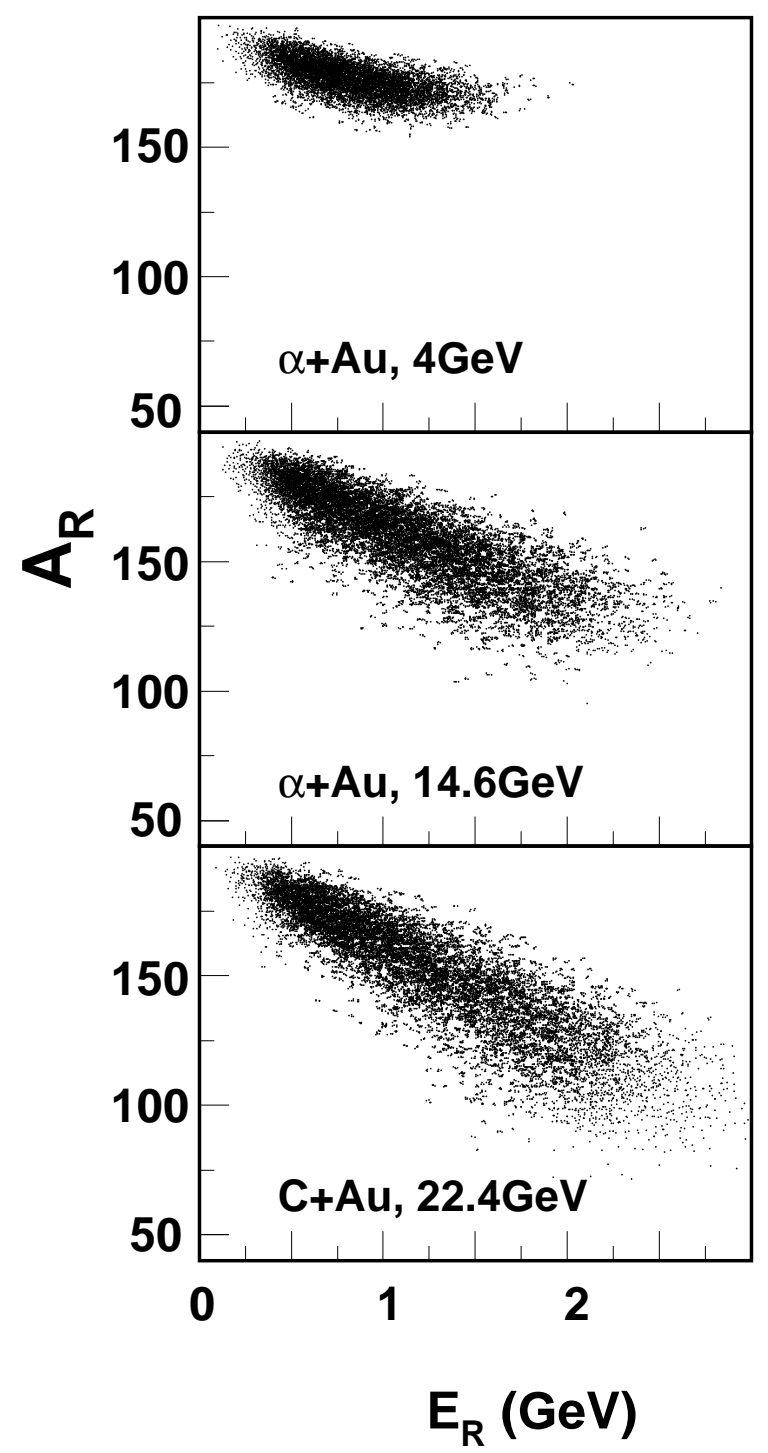

Fig. 2. Distribution of residual masses $A_{R}$ and excitation energies $E_{R}$ after the cascade calculation for ${ }^{4} \mathrm{He}+\mathrm{Au}$ at 4 and $14.6 \mathrm{GeV},{ }^{12} \mathrm{C}+\mathrm{Au}$ at $22.4 \mathrm{GeV}$. 


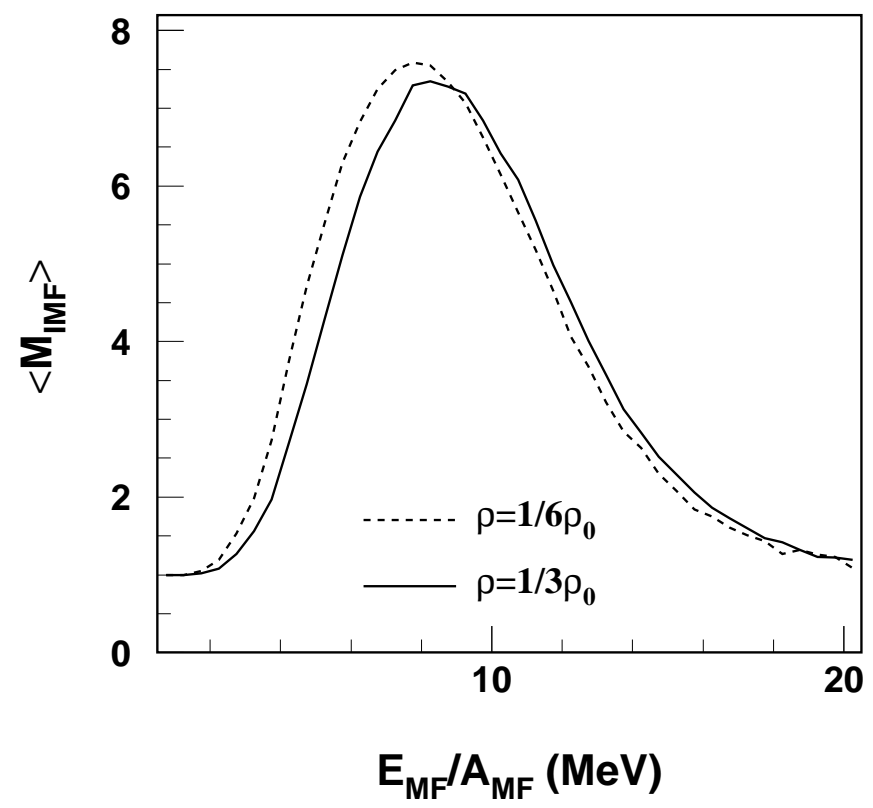

Fig. 3. Mean IMF multiplicities (for events with at least one IMF) as a function of the thermal excitation energy according to SMM, calculated for freeze-out densities $\approx 1 / 3 \rho_{0}$ and $1 / 6 \rho_{0}$. 


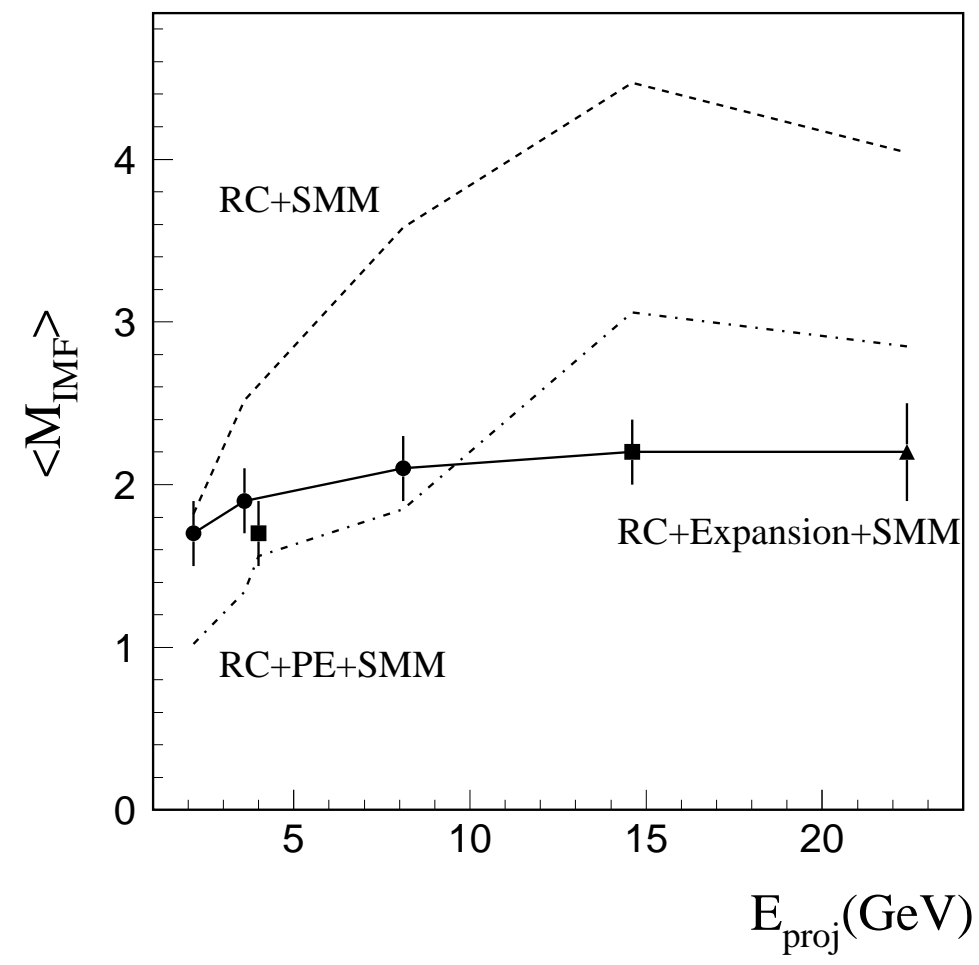

Fig. 4. Mean IMF multiplicities (for events with at least one IMF) as a function of beam energy. The points are experimental data(circles - for p; squares - for ${ }^{4} \mathrm{He}$, triangles - for ${ }^{12} \mathrm{C}$. Dashed and dash-dotted lines are drawn through the values calculated with $\mathrm{RC}+\mathrm{SMM}$ and with $\mathrm{RC}+\mathrm{PE}+\mathrm{SMM}$ at the beam energies used. The solid line is obtained with the use of $\mathrm{RC}+\alpha+\mathrm{SMM}$. For simplicity, only one line is drawn for a given model calculation neglecting some dependence on projectile mass. 

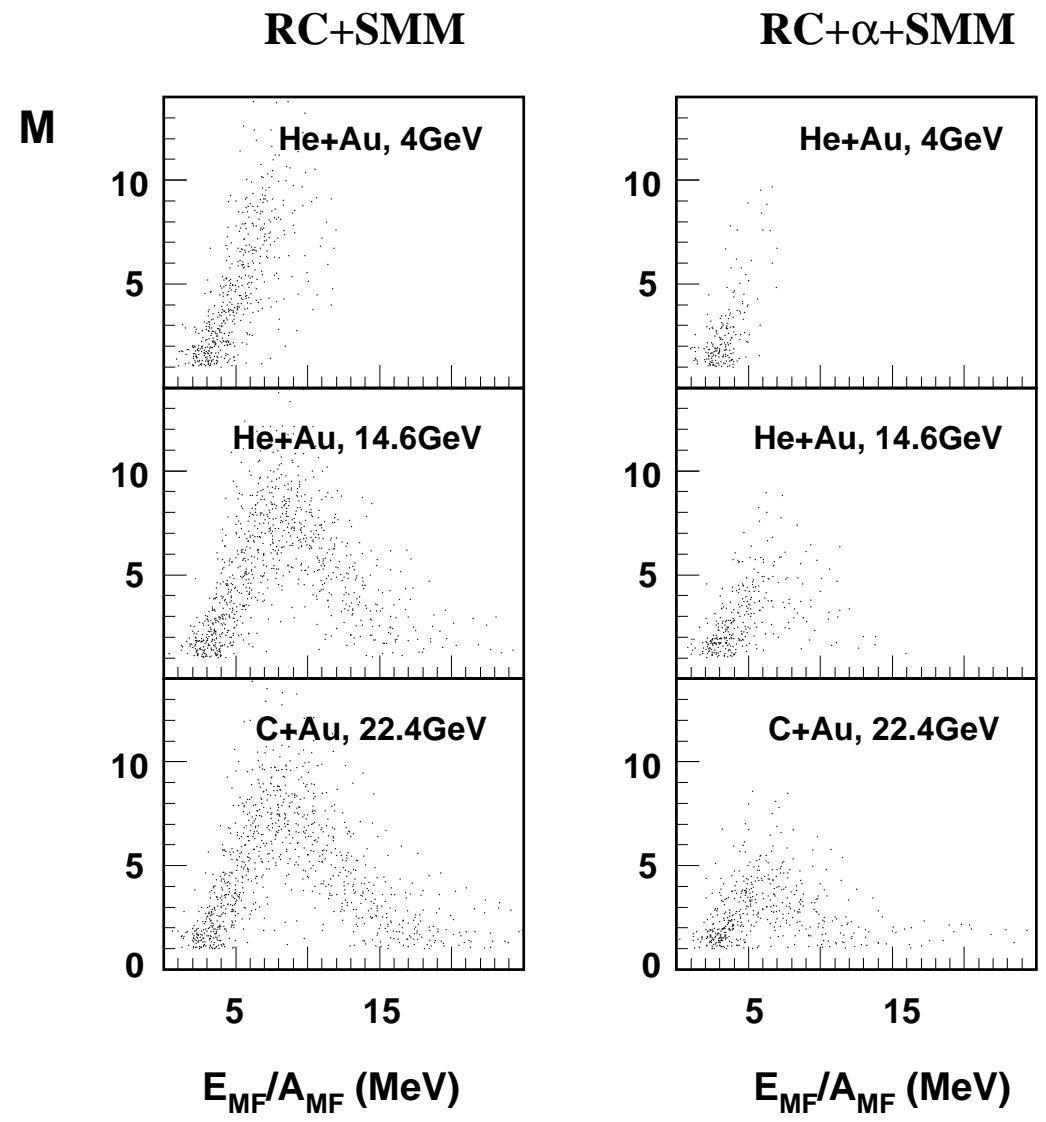

Fig. 5. The calculated event distribution in $M-E_{M F} / A_{M F}$ plane: $\mathrm{RC}+\mathrm{SMM}$ model (left) and $\mathrm{RC}+\alpha+\mathrm{SMM}$ approach (right). 


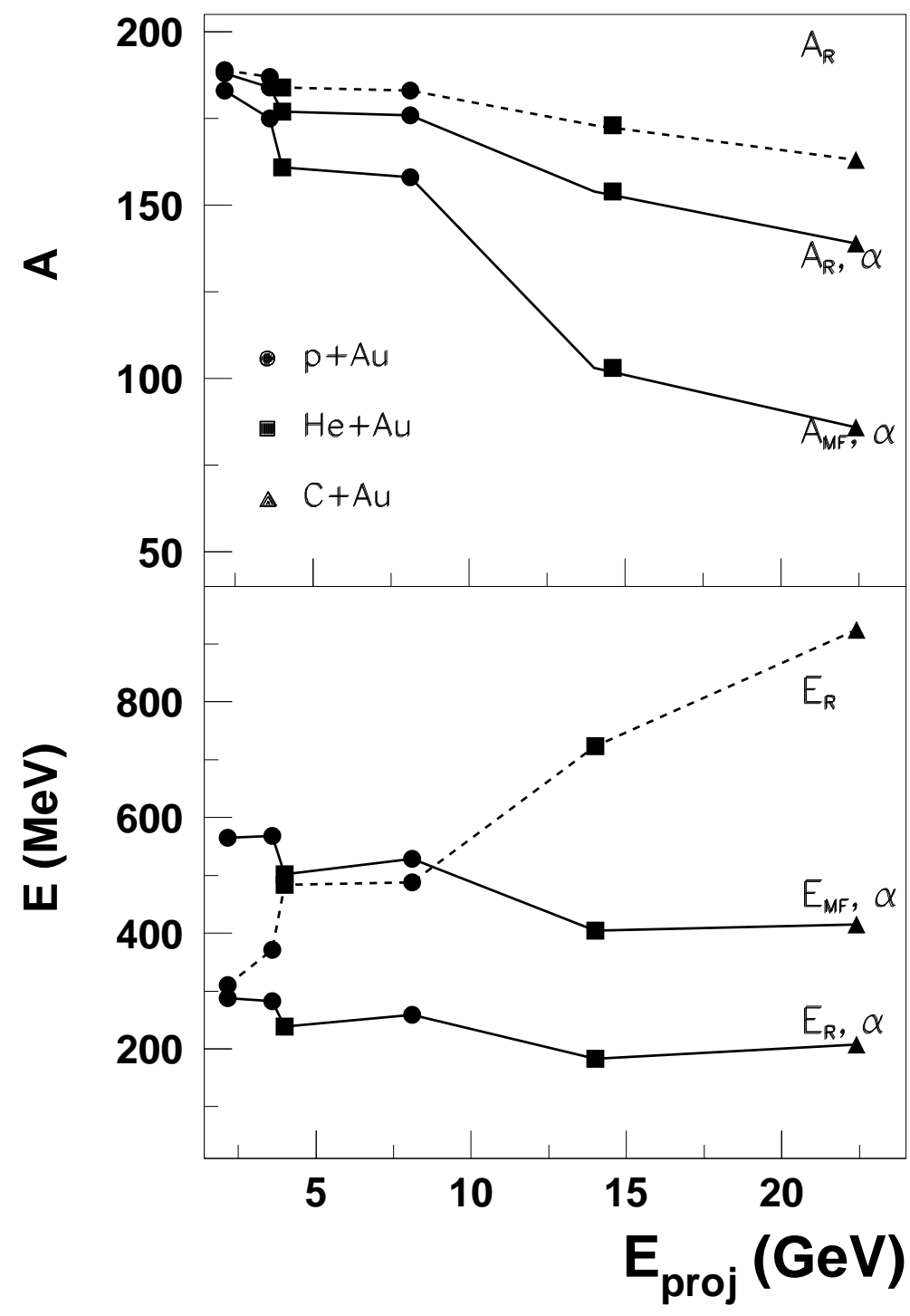

Fig. 6. The mean values of the remnant excitation energies and mass numbers according to Table 1: $E_{R}$ and $A_{R}$ are averaged over all inelastic collisions, $E_{M F}$, $A_{M F}$ are for fragmenting residues. The calculations with $\mathrm{RC}+\alpha+\mathrm{SMM}$ are marked by " $\alpha$ ". 


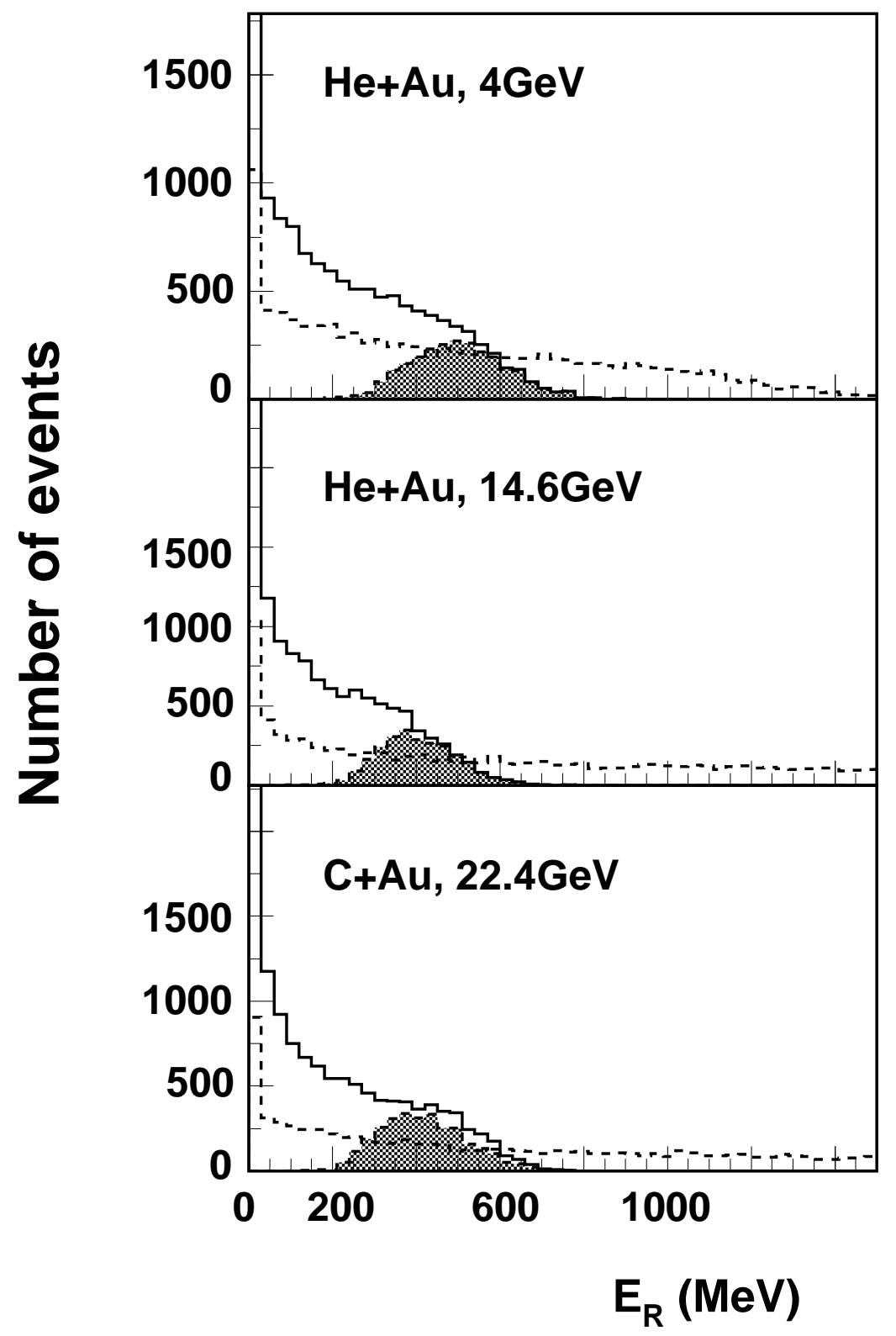

Fig. 7. Distribution of excitation energies obtained with RC (dashed lines), with $\mathrm{RC}+\alpha$ (solid lines) and the fraction decaying by multifragmentation according to $\mathrm{RC}+\alpha+\mathrm{SMM}$ (hatched area). 

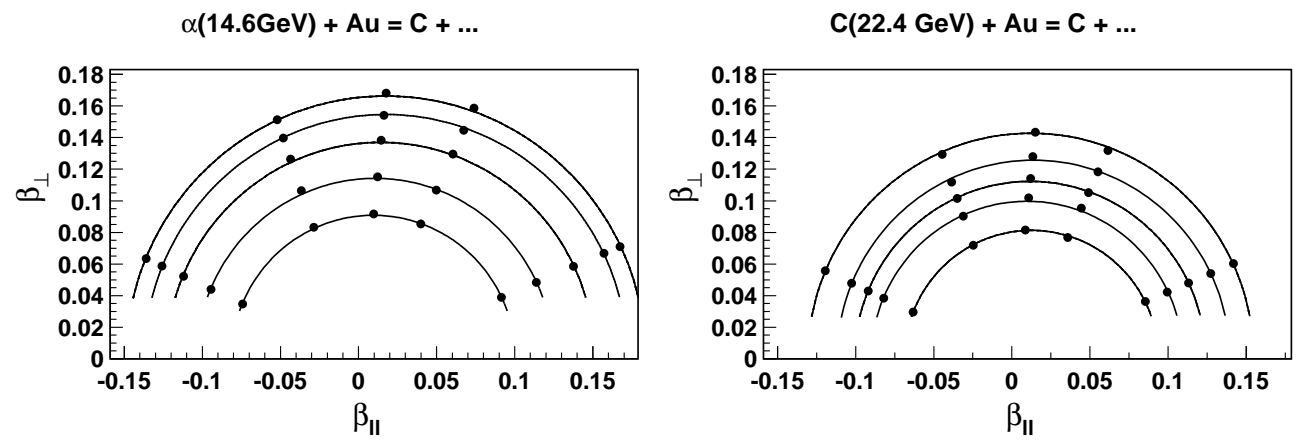

Fig. 8. Transverse versus longitudinal velocity plot for emitted carbon isotopes produced in ${ }^{4} \mathrm{He}(14.6 \mathrm{GeV})$ and ${ }^{12} \mathrm{C}(22.4 \mathrm{GeV})$ collisions with a $\mathrm{Au}$ target. Circles are drawn through points of equal invariant cross section corresponding to isotropic emission of the fragments in the moving source frame.

\section{proj. $+\mathrm{Au}=\mathrm{C}+\ldots$}

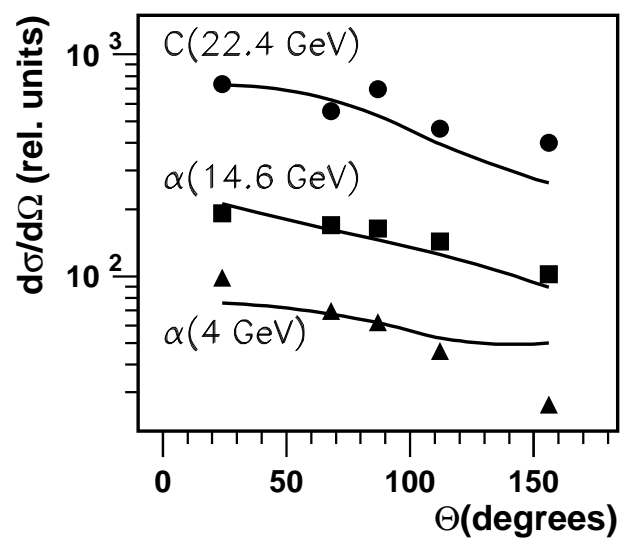

Fig. 9. Angular distributions of carbon (in laboratory system) for ${ }^{4} \mathrm{He}+\mathrm{Au}$ and ${ }^{12} \mathrm{C}+\mathrm{Au}$ collisions. The lines are calculated with $\mathrm{RC}+\alpha+\mathrm{SMM}$. 

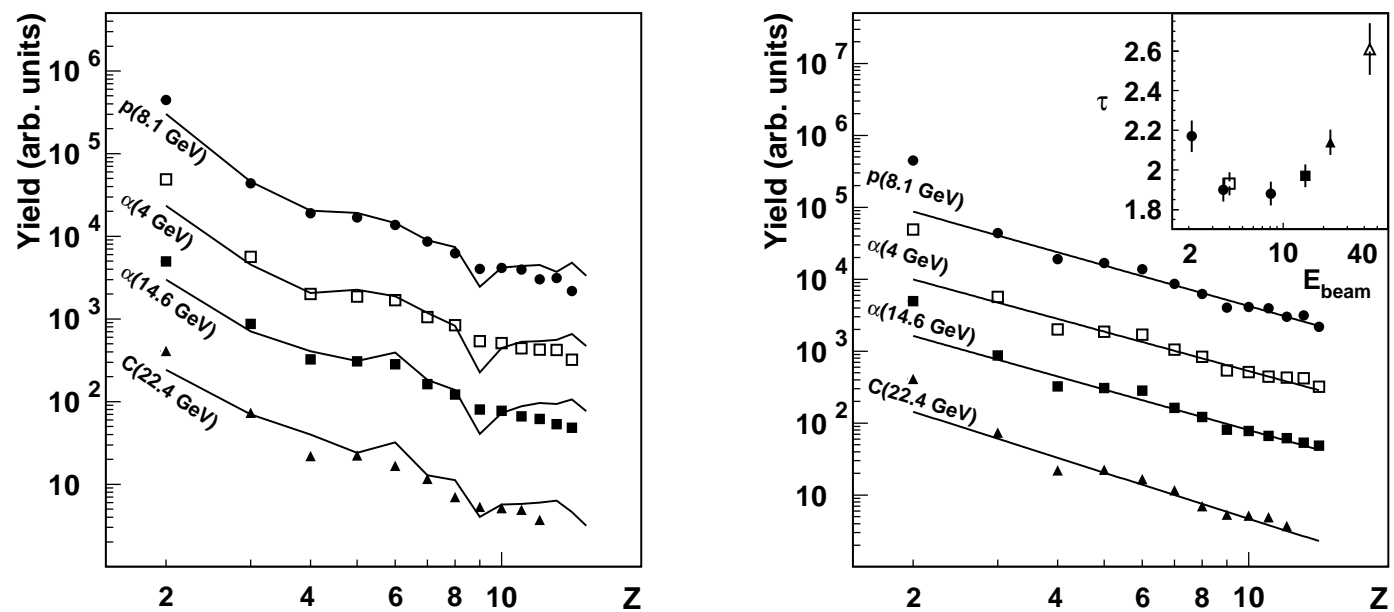

Fig. 10. Fragment charge distributions obtained at $\theta=87^{\circ}$ for $\mathrm{p}+\mathrm{Au}$ at $8.1 \mathrm{GeV}$ (top), ${ }^{4} \mathrm{He}+\mathrm{Au}$ at $4 \mathrm{GeV},{ }^{4} \mathrm{He}+\mathrm{Au}$ at $14.6 \mathrm{GeV}$ and ${ }^{12} \mathrm{C}+\mathrm{Au}$ at $22.4 \mathrm{GeV}$. The lines (left side) are calculated by $\mathrm{RC}+\alpha+\mathrm{SMM}$ (normalized at $Z=3$ ). The power law fits are shown on the right panel with $\tau$-parameters given in the insert as a function of beam energy. The last point in the insert is for ${ }^{12} \mathrm{C}+\mathrm{Au}$ collisions at $44 \mathrm{GeV}$ (from a preliminary experiment).

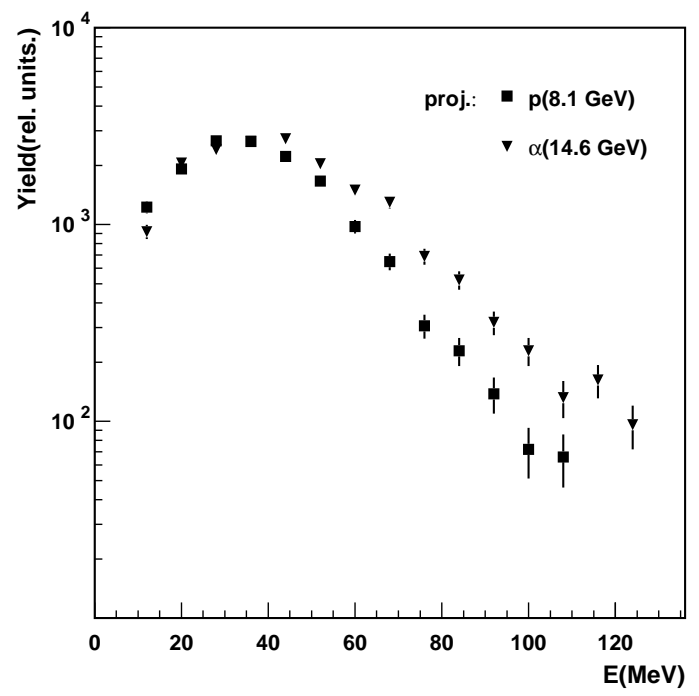

Fig. 11. Comparison of energy spectra of carbon fragments from $\mathrm{p}+\mathrm{Au}$ at $8.1 \mathrm{GeV}$ and $\alpha+\mathrm{Au}$ at $14.6 \mathrm{GeV}$. 


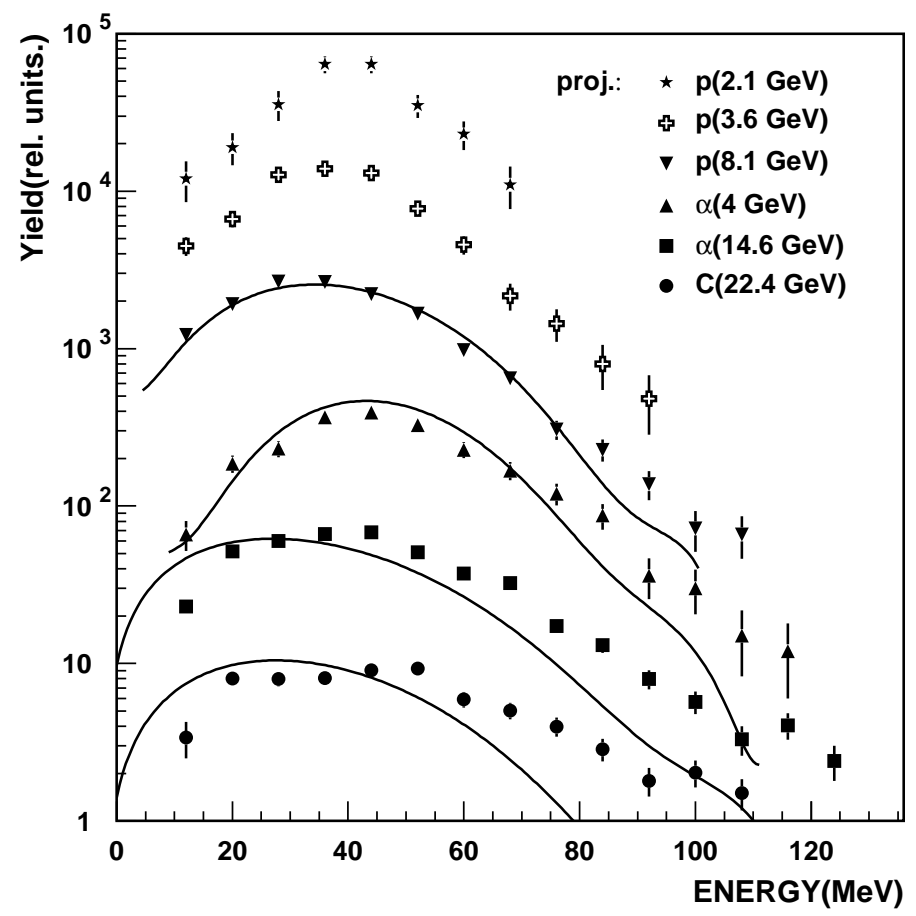

Fig. 12. Energy distribution of carbon fragments obtained for different collision systems at $\theta=87^{\circ}$. The lines are calculated in $\mathrm{RC}+\alpha+\mathrm{SMM}$ model assuming no flow. 


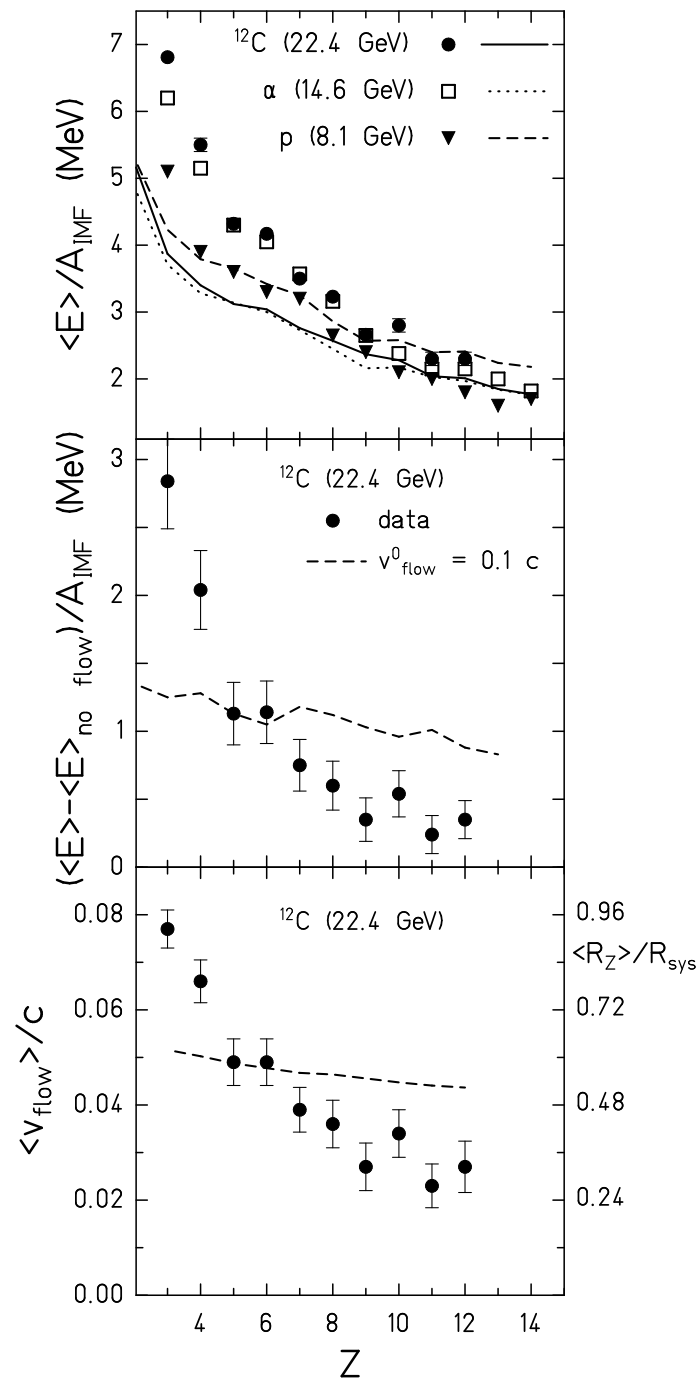

Fig. 13. Upper part: Mean kinetic energies of outgoing fragments per nucleon measured at $\theta=87^{\circ}$ for $\mathrm{p}(8.1 \mathrm{GeV}),{ }^{4} \mathrm{He}(14.6 \mathrm{GeV})$ and ${ }^{12} \mathrm{C}(22.4 \mathrm{GeV})$ collisions with $\mathrm{Au}$. The lines are calculated within $\mathrm{RC}+\alpha+\mathrm{SMM}$ approach assuming no flow. Middle part: Flow energy per nucleon (dots) obtained as a difference of the measured fragment kinetic energies and the values calculated under assumption of no flow in the system. The dashed line represents a calculation assuming a linear radial profile for the expansion velocity with $v_{\text {flow }}^{0}=0.1 \mathrm{c}$. Lower part: Experimentally deduced mean flow velocities (dots) for ${ }^{12} \mathrm{C}+\mathrm{Au}$ collisions as a function of the fragment charge (left scale), and the mean relative radial coordinates of fragments (right scale), obtained under assumption of a linear radial profile for the expansion velocity. The dashed line shows the mean radial coordinate according to SMM. 


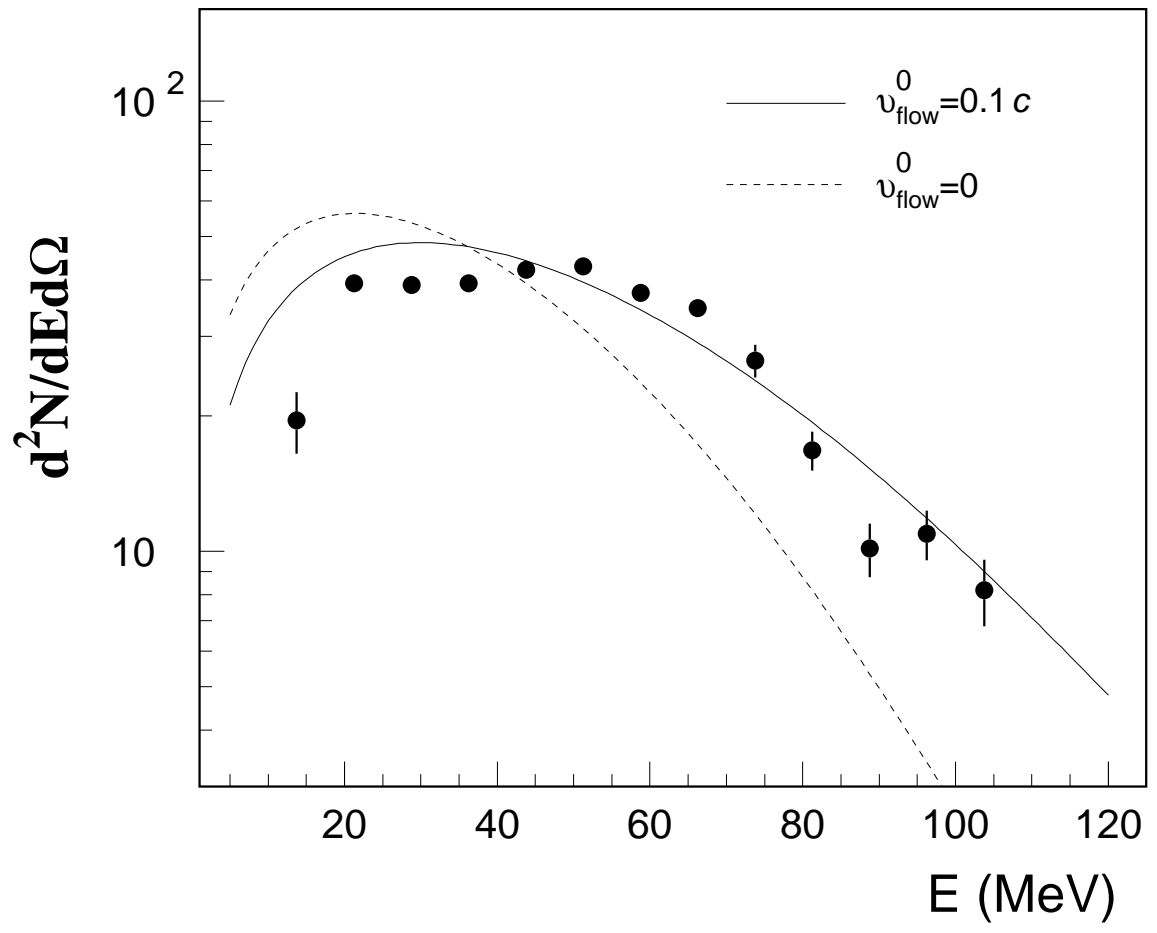

Fig. 14. Energy distribution of carbon fragments $\left(\right.$ at $\theta=87^{\circ}$ ) from ${ }^{12} \mathrm{C}+\mathrm{Au}$ collisions. Solid line is calculated assuming the radial flow with velocity on the surface equal to 0.1c. Dashed line is calculated assuming no flow. 


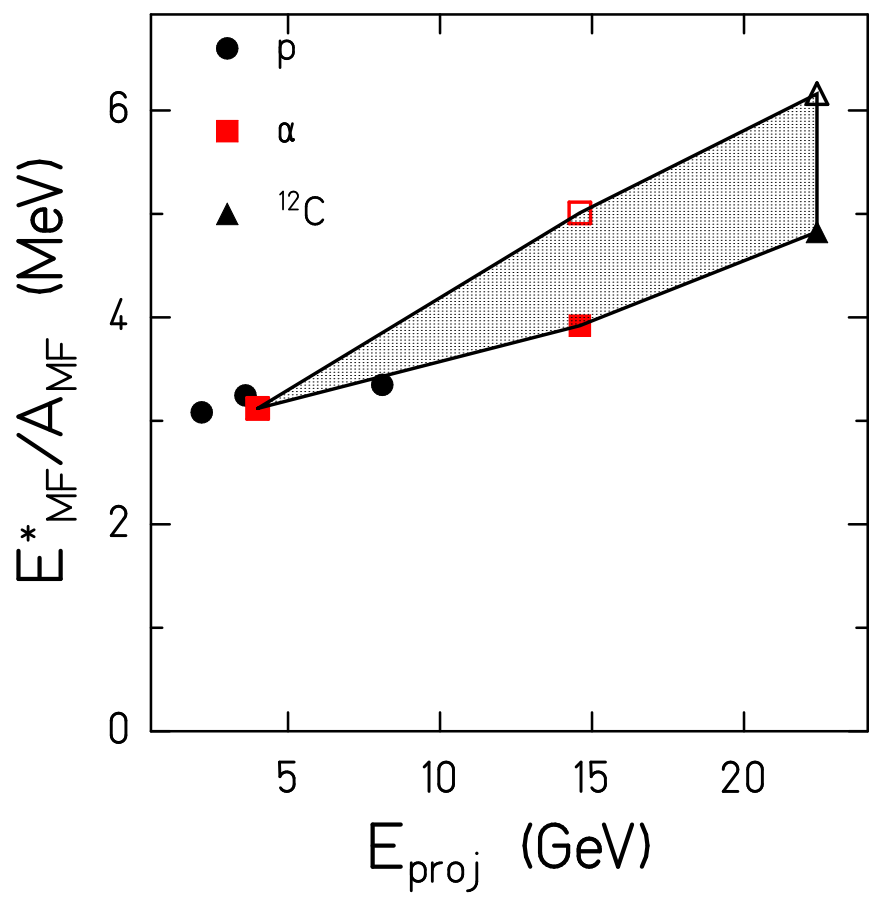

Fig. 15. Mean excitation energy of the fragmenting nucleus per nucleon $E_{M F}^{*} / A_{M F}$ as a function of the beam energy: the solid points refer to the thermal part, in some cases the flow energy added is shown as open symbols and grey area. 
$\mathbf{R C}+\mathbf{S M M}$

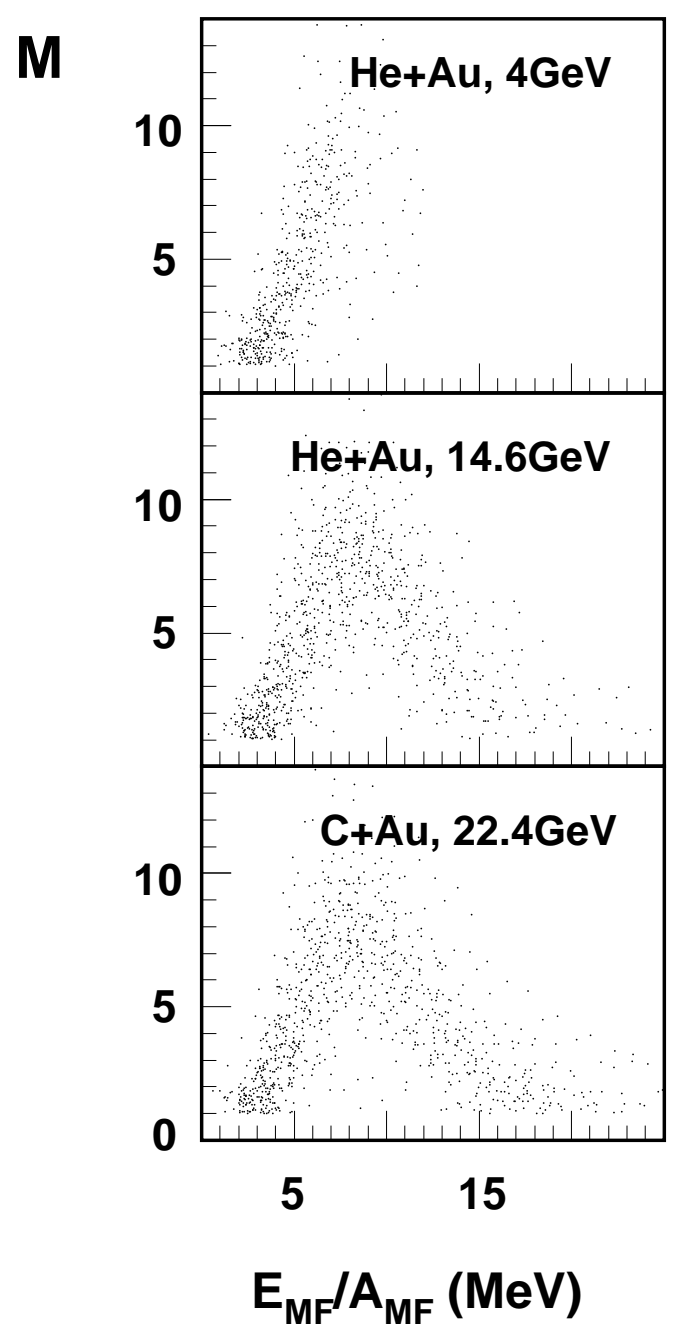

$\mathbf{R C}+\alpha+\mathbf{S M M}$

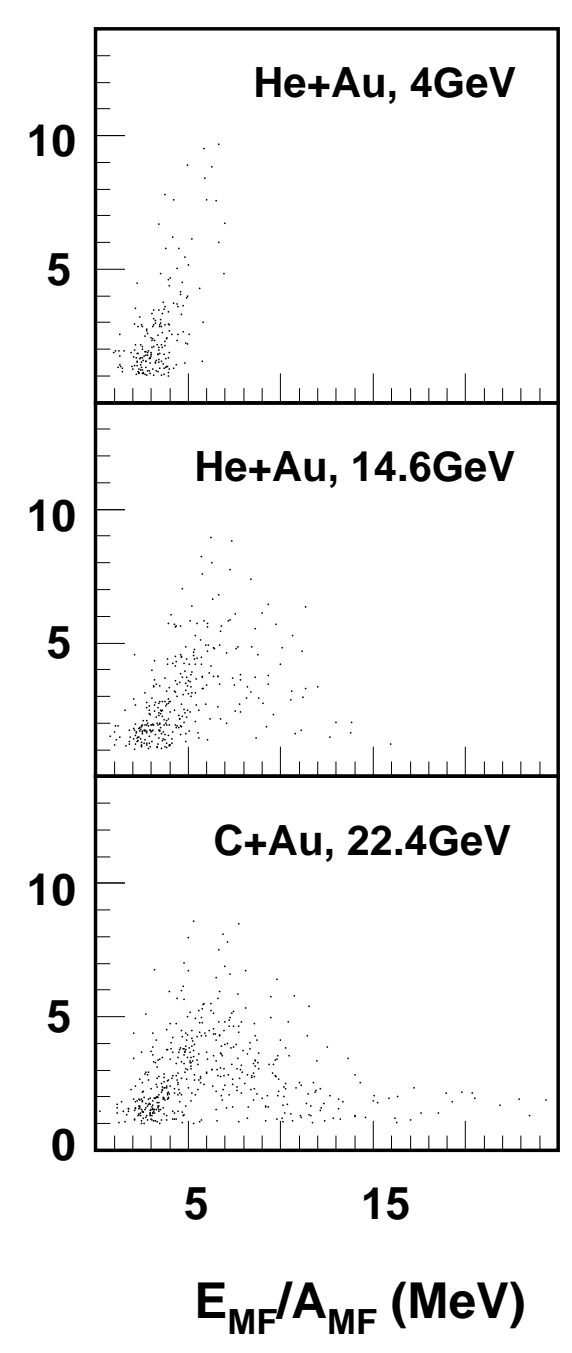

